This document is the Accepted Manuscript version of a Published Work that appeared in final form in Langmuir 201935 (47), 15168-15179, copyright ( 2019 American Chemical Society after peer review and technical editing by the publisher. To access the final edited and published work see https://doi.org/10.1021/acs.langmuir.9b02215

\title{
How confinement affects the nucleation, crystallization and dielectric relaxation of poly(butylene succinate) and poly(butylene adipate) infiltrated within nanoporous alumina templates
}

\author{
Maryam Safari ${ }^{1}$, Jon Maiz ${ }^{1}$, Guangyu Shi ${ }^{2,6}$, Diana Juanes ${ }^{3}$, Guoming Liu ${ }^{2,6}$, Dujin Wang ${ }^{2,6}$, \\ Carmen Mijangos*3,4, Ángel Alegría ${ }^{4}$, Alejandro J. Müller $* 1,5$
}

\begin{abstract}
${ }^{1}$ POLYMAT and Polymer Science and Technology Department, Faculty of Chemistry, University of the Basque Country UPV/EHU, Paseo Manuel de Lardizábal, 3, 20018 Donostia-San Sebastián, Spain.
\end{abstract}

${ }^{2}$ Beijing National Laboratory for Molecular Sciences, CAS Key Laboratory of Engineering Plastics, CAS Research/Education Center for Excellence in Molecular Sciences, Institute of Chemistry, the Chinese Academy of Sciences, Beijing 100190, China.

${ }^{3}$ Instituto de Ciencia y Tecnología de Polímeros, Consejo Superior de Investigaciones Científicas, ICTP-CSIC, Juan de la Cierva 3, Madrid 28006, Spain.

${ }^{4}$ Departamento de Física de Materiales, University of the Basque Country UPV/EHU and Centro de Física de Materiales (CFM) (CSIC-UPV/EHU) - Materials Physics Center (MPC), Paseo Manuel de Lardizabal 5, 20018 San Sebastián, Spain.

\footnotetext{
${ }^{5}$ IKERBASQUE, Basque Foundation for Science, Bilbao, Spain.

${ }^{6}$ University of Chinese Academy of Sciences, Beijing 100049, China.
}

*Corresponding author: alejandrojesus.muller@ehu.es cmijangos@ictp.csic.es 


\section{ABSTRACT}

This work describes the successful melt infiltration of poly(butylene succinate) (PBS) and poly(butylene adipate) (PBA) within $70 \mathrm{~nm}$ diameter anodic aluminum oxide (AAO) templates. The infiltrated samples were characterized by SEM, Raman, and FTIR spectroscopy. The crystallization behavior and crystalline structure of both polymers, bulk and confined, were analyzed by differential scanning calorimetry (DSC) and grazing incidence wide angle x-ray scattering (GIWAXS). DSC revealed that a change in the nucleation process occurred from heterogeneous nucleation for bulk samples, to homogeneous nucleation for infiltrated PBA, and to surface-induced nucleation for infiltrated PBS. GIWAXS results indicate that PBS nanofibers crystallize in the $\alpha$-phase, as well as their bulk samples. However, PBA nanofibers crystallize just in the $\beta$-phase, whereas PBA bulk samples crystallize in a mixture of $\alpha$ - and $\beta$-phases. The crystal orientation within the pores was

determined, and differences between PBS and PBA were also found. Finally, broadband dielectric spectroscopy (BDS) was applied to study the segmental dynamics for bulk and infiltrated samples. The glass temperature was found to significantly decrease in the PBS case upon infiltration while that of PBA remained unchanged. These differences were correlated with the higher affinity of PBS to the AAO walls as compared to PBA, in accordance with their nucleation behavior (surface-induced versus homogeneous nucleation respectively).

Keywords: polymer infiltration, AAO template, PBA, PBS, Dielectric spectroscopy 


\section{INTRODUCTION}

Poly(butylene succinate) (PBS) and poly(butylene adipate) (PBA) are biodegradable commercial synthetic polyesters. These polymers have similar properties to linear low-density polyethylene and can be processed in conventional equipment commonly used for polyolefins. ${ }^{1}$ This is the reason, why the applications of these biopolyesters have found a place in the market. Nevertheless, although from a macroscopic point of view, polymer properties of both polyesters have already been studied, their properties at the nanoscale level have been scarcely reported, even when it is known that confining a polymeric material into nanosized cavities introduces a new variable to those determining the properties of the material. In fact, the nucleation and crystallization of semi-crystalline polymers are dramatically affected by confinement ${ }^{2-7}$ and also segmental, and molecular dynamics are hindered or restricted in many polymers, by confinement. ${ }^{8-11}$ Moreover, when confining polymers in nanocavities, polymer-wall, and polymer-substrate interactions can give rise to adsorption and/or to the formation of different chain conformations that will affect both structural and dynamic properties. So, it seems crucial to determine how polymer properties, crystallization and polymer dynamics of PBS and PBA can be affected by confinement, in particular, in cylindrical Anodic Aluminum Oxide (AAO) templates, one of the most studied spatial confinement systems.

Tailored anodic aluminum oxide templates fabricated by a two anodization procedure ${ }^{12-}$

16, contain arrays of aligned cylindrical nanopores with narrow pore diameter distributions. Nanocavities of AAO templates provide versatility with respect to the diameter and length of the pores that are not interconnected. As reported in the literature, self-ordered nanoporous anodic aluminum oxide templates have been largely employed in the preparation of polymer nanofibers and nanotubes with diameters ranging from 20 to $400 \mathrm{~nm}$ and lengths from $700 \mathrm{~nm}$ to higher than 
100 microns. Thus, AAO nanocavities have been infiltrated by many polymers and polymerbased composites of different chemical nature and their polymer properties/structure relationship studied as a function of the degree of confinement.

Two of the most relevant physical phenomena studied in relation to the final properties of a polymer in confinement are crystallization and polymer dynamics. Moreover, crystalline regions can significantly modify the relaxation behavior of polymer chains in the amorphous regions and consequently affect many important physical properties. Confinement effects on polymer crystallinity and crystal structure and molecular dynamics of semicrystalline polymers, such as, poly(ethylene) oxide (PEO) ${ }^{3}, 17$, poly(E-caprolactone) (PCL) ${ }^{4}{ }^{18}$, poly(vinylidene fluoride) $\quad(\mathrm{PVDF})^{19-21}, \quad$ poly(L-lactide $) \quad(\mathrm{PLLA})^{22}, \quad$ polypropylene(PP) ${ }^{16}, \quad$ poly(3hydroxybutyrate)(PHB) ${ }^{23}$, poly(ethylene)(PE) $)^{24}$, syndiotactic polystyrene (sPS) ${ }^{25}$, poly(3dodecylthiophene) (P3DDT) ${ }^{26}$, Nylon- $6^{27}$, and even block copolymers with PEO and PE crystallizable blocks ${ }^{24}$, just to name a few examples, have been studied in the past.

Recent reviews on polymer crystallization discuss AAO infiltrated systems, as well as, polymer confined in droplets, ultrathin films or nanolayers. ${ }^{7,28-31}$ One of the main conclusions of these works is that, as confinement increases, the crystallization temperature can experience large decreases, reflecting a change from heterogeneous nucleation in the bulk polymer to surface or homogeneous nucleation for polymers infiltrated inside nanopores. In some cases, fractionated crystallization has been reported (see references 28 and 29 and references therein). Recently, some of us reported ${ }^{18}$ that if a proper removal of the polymer layer that typically remains after infiltration is not achieved, this leads to pore connection at the surface of the AAO. In this case, such percolation of pores by a polymer layer can cause the appearance of fractionated crystallization. It has also been reported, that the crystallization of homogeneously nucleated crystals decreases as pore volume decreases (i.e., increasing confinement) since the probability 
of nucleation also decreases and if the pore volume is below a critical size (around $10 \mathrm{~nm}$ ), crystallization can be totally inhibited. ${ }^{2}$

In summary, nanoconfinement plays a significant role in two main factors of crystallization: (a) a change in nucleation mechanism, from heterogeneous nucleation to surface or homogeneous nucleation and (b) the dependence of the crystallization temperature on the volume or the surface (or interphase) of the crystallizable micro or nanodomains. Moreover, the melting point also decreases with confinement but to a much lesser degree than the crystallization temperature. Also, Su et al. reported a preferential orientation of polymeric crystals generally induced by one or two-dimensional confinement. ${ }^{32}$ Avrami indexes decrease with confinement until values of 1 (or even lower) are achieved in the limit of isolated domains, as the material approaches a first-order (or lower) crystallization kinetics. This type of kinetics reflects that nucleation is the rate-determining step in the overall crystallization of ideally confined polymers. ${ }^{7}$, 28-30, 33

The molecular motions in polymer systems can be easily investigated using neutron diffraction and relaxation techniques. ${ }^{34-35}$ Both techniques allow monitoring glass transition changes in confined situations in comparison to the bulk material. Confinement effects are of two types, pure confinement effects due to the hindering of a molecular motion, as a consequence of restricted space, and associated confinement effects, i.e., they are not a direct consequence of a confined molecular motion but related to surface interaction effects and, therefore, also related to low dimensionality, although they can often be masked. Nevertheless, this effect cannot be underestimated since, in many nanomaterials applications, the polymer is close to a surface which effectively confines it. In this kind of materials, surfaces are usually attractive, so interactions take place. 
Recently, it has been shown that confinement not only affects segmental dynamics but also the chain dynamics of polymers, where an unexpected enhancement of the flow and a reduction in intermolecular entanglements has been found. Only to cite some examples, the influence of polymer confinement within templates of cylindrical nanopores on the single-chain dynamics of PEO has been studied by neutron scattering techniques, ${ }^{3,17,36}$, and the segmental dynamics and the relaxation behavior of PVDF ${ }^{19}$, cis-1,4 polyisoprene ${ }^{10}, \mathrm{PHB}^{23}$ and PMMA by broad band dielectric spectroscopy. ${ }^{37}$ Results showed that the properties of the infiltrated polymers largely differ from those of the respective bulk polymer. However, the influence of confinement on the dynamic properties of polymer nanostructures is far from generalized and needs to be studied for each particular case, i.e., for PBS and PBA, among other biopolymers.

Taking into account previous results for semicrystalline polymers ${ }^{19,}{ }^{36}$, there is not a general rule that can predict how confinement affects the properties of semicrystaline polymers. In fact, changes in nucleation mechanisms depend on the specific polymers under study and it is very difficult to predict if the nucleation will change from heterogeneous in the bulk to homogeneous or surface-nucleated under strict hard confinement cases. Therefore, the necessity of a specific study for each type of polymer under confinement arises, in order to contribute to the general understanding of polymers under confinement. The present work investigates the influence of confinement effects on dielectric and thermal properties of PBS and PBA infiltrated within nanoporous AAO templates. Both PBA and PBS are biodegradable aliphatic polyesters with a crystallization degree in the range $35-45 \%$ and glass transition temperatures, $T_{g}$, of around $-55^{\circ} \mathrm{C}$ and $-35^{\circ} \mathrm{C}$, respectively. The samples were studied by Differential Scanning Calorimetry (DSC), Grazing Incidence Wide Angle X-Ray Scattering (GIWAXS), Scanning Electron Microscope (SEM), Broadband Dielectric Spectroscopy (BDS), Infrared (IR) and Raman spectroscopy. Very large differences were found in the nucleation and crystallization of the 
infiltrated materials with respect to the polymers in bulk. The dielectric relaxation results indicated that molecular relaxations of the amorphous parts of both PBS and PBA were also significantly affected by confinement.

\section{EXPERIMENTAL SECTION}

\section{Materials and methods}

Fabrication of AAO templates. The homemade AAO templates have been fabricated using the well-known two-step electrochemical anodization process of aluminum foils ${ }^{12}$, which has been previously described in detail. ${ }^{12,38-39}$ Previously, the aluminum was cleaned by sonication in different solvents that have different polarity (acetone, 2-propanol, water, and ethanol). Subsequently, the foils undergo a 4 min electropolishing process in a solution of perchloric acid and ethanol (1/3) with a constant voltage of $20 \mathrm{~V}$ and a temperature below $283 \mathrm{~K}$. Firstly, AAO templates of $35 \mathrm{~nm}$ pore diameter and $30 \mu \mathrm{m}$ in length were synthesized. For the first electrochemical anodization process, oxalic acid was used as an electrolyte in a concentration of $0.03 \mathrm{M}$. Anodization time was 19 hours at 270.5 K. For the second electrochemical anodization process to length the nanopores, similar conditions were used. Subsequently, a process of widening of the pores in $5 \% \mathrm{H}_{3} \mathrm{PO}_{4}$ was carried out until a size of $70 \mathrm{~nm}$ pore diameter was reached. The AAO templates are opened on one side only. The other side is closed and also contains the original aluminum base employed for the preparation of the template.

Poly (butylene succinate) with $M_{w}=15000 \mathrm{~g} / \mathrm{mol}$ and a glass transition temperature of $T_{g}$ $=243 \mathrm{~K}$, was supplied by Aldrich. Poly (butylene adipate) with $M_{w}=12000 \mathrm{~g} / \mathrm{mol}$ and $T_{g}=218$ $\mathrm{K}$, was purchased from Aldrich. 
Samples preparation by polymer infiltration in AAO templates. The infiltration of poly (butylene succinate) and poly (butylene adipate) was carried out by the melt precursor film wetting method. Polymer films were employed and placed on the AAO template surface and then infiltrated by heating them to $453 \mathrm{~K}$ for $12 \mathrm{~h}$ and $373 \mathrm{~K}$ for $6 \mathrm{~h}$ under nitrogen flow, for PBS and PBA respectively. Later, samples were quenched to room temperature and carefully cleaned to remove any residual polymer from the template surface, closely following the procedure recommended by Shi et al. ${ }^{18}$

\section{Morphological and chemical characterization}

Scanning Electron Microscopy (SEM). Philips XL-30 ESEM was used to morphologically characterize the AAO templates and confined polymer samples. To observe the polymer nanofibers by SEM, alumina substrates were dissolved with $\mathrm{NaOH} 1 \mathrm{M}$ during $1 \mathrm{~h}$ at room temperature (RT).

Chemical characterization by Raman Spectroscopy (RAMAN) and Fourier Transformed Infrared Spectroscopy (FTIR). Raman spectroscopy was applied to study and verify the effectiveness of the infiltration of PBA and PBS. Renishaw InVia Raman Microscope (Renishaw plc, Wottonunder-Edge, UK) was used fitted with a grating spectrometer of $785 \mathrm{~nm}$ near-infrared diode laser and a Peltier-cooled charge-coupled device (CCD) detector, attached to a confocal microscope. All spectra were processed using Renishaw WiRE 3.3 software. Data acquisition covered the spectral range $3000-200 \mathrm{~cm}^{-1}$ with a spectral resolution of $4 \mathrm{~cm}^{-1}$. 
To verify if any conformational changes happened during the infiltration processes, polymer nanofibers were examined by Fourier transform infrared spectroscopy (FTIR) employing a PerkinElmer Spectrum One coupled with an attenuated total reflectance (ATR) accessory.

\section{Crystallization process and crystalline structure characterization}

Crystallization process. The non-isothermal crystallization behavior of bulk and infiltrated polymers was examined by differential scanning calorimetry (DSC) using a DSCQ2000, TA calorimeter under a nitrogen atmosphere flow. Indium was used as a standard for calibration. Samples were heated from room temperature to $30 \mathrm{~K}$ above their melting point and held at this temperature for 3 min to erase thermal history. Then, they were cooled to $213 \mathrm{~K}$ at 10 $\mathrm{K} / \mathrm{min}$ and subsequently heated above their melting point. Around $5 \mathrm{mg}$ of bulk polymer and 15 mg of confined samples inside AAO were sealed in standard aluminum pans and samples were measured with aluminum base. Previous to DSC measurements, after melt infiltration, and in order to remove the excess polymer on the AAO surfaces, templates were cleaned in four steps, as follows: 1. Removing the excess bulk polymer from the surface using a sharp blade at room temperature, 2. Samples were further cleaned with a soft cloth at a temperature above the melting temperature of the polymer (413 K for PBS and $373 \mathrm{~K}$ for PBA), 3. The samples were polished using fine grade sandpaper, 4. Finally, the surface of the templates was cleaned with chloroform.

$2 D$ Wide-Angle X-ray Diffraction (WAXD). The 2D wide-angle X-ray diffraction (WAXD) measurements were performed at $25^{\circ} \mathrm{C}$ on a Xeuss 2.0 SAXS/WAXS system (Xenocs $\mathrm{SA}$, France). The $\mathrm{Cu} \mathrm{K} \alpha \mathrm{X}$-ray source (GeniX3D Cu ULD), generated at $50 \mathrm{kV}$ and $0.6 \mathrm{~mA}$, was utilized to produce X-ray radiation with a wavelength of $1.5418 \AA$. A semiconductor detector 
(Pilatus 300K, DECTRIS, Swiss) with a resolution of $487 \times 619$ pixels (pixel size $=172 \times 172$

$\mu \mathrm{m}^{2}$ ) was used to collect the scattering signals. The X-rays irradiate the sample along the $\mathrm{x}$-axis with an incident angle of $3^{\circ}$ with respect to the AAO surface. The 2D detector was placed perpendicular to the incident beam. The exposure time for each pattern was 20 min for infiltrated samples and 5 min for bulk samples. The one-dimensional intensity profiles were integrated from the 2D WAXS patterns and averaged along the azimuthal angle. Samples were treated before the measurements using a Linkam hot-stage to: 1 . erase thermal history at $30 \mathrm{~K}$ above their melting point; 2. Cooldown to $213 \mathrm{~K}$ at $5 \mathrm{~K} / \mathrm{min}$. Then, the samples were stored at room temperature, and the measurements were made at room temperature.

\section{Dielectric Relaxation Processes}

The complex dielectric permittivity $\varepsilon^{*}(\omega)=\varepsilon^{\prime}(\omega)-i \varepsilon^{\prime \prime}(\omega)$, where $\varepsilon^{\prime}$ is the real and $\varepsilon^{\prime \prime}$ is the imaginary part, was obtained as a function of the frequency $\omega$ and temperature $T$ by using a Novocontrol high-resolution dielectric analyzer (Alpha-N analyzer). The sample cell was set in a cryostat, and its temperature was controlled via a nitrogen gas jet stream coupled with the Novocontrol Quatro controller. The dielectric measurements were performed at different temperatures in the range of $120 \mathrm{~K}$ and $390 \mathrm{~K}$ and at frequencies in the range of $10^{-1}-10^{6} \mathrm{~Hz}$. For PBA and PBS homopolymer samples, measurements were carried out in the usual parallel plate geometry with electrodes of $20 \mathrm{~mm}$ in diameter. A separation of $100 \mathrm{~mm}$ between both electrodes was maintained by using a cross-shaped Teflon ${ }^{\circledR}$ spacer of a small area. For the samples infiltrated in the AAO templates, the measurements were carried out with a $15 \mathrm{~mm}$ electrode placed on top of the template without spacers. Before the dielectric measurements, the sample 
capacitor (inside the BDS cell) was heated above the melting temperature of each polymer to erase the thermal history.

\section{RESULTS AND DISCUSSION}

\section{Sample preparation and SEM observations}

SEM allows observing the surface of the AAO templates and the obtained nanofibers, after the partial dissolution of the alumina template with $1 \mathrm{M} \mathrm{NaOH}$ solution. Figure 1a shows a representative image of the manufactured AAO templates, which confirms the size of the nanopores of the obtained templates. The pore size distribution on the sample surface is uniform with very well-defined geometry. Figures $1 \mathrm{~b}$ and $1 \mathrm{c}$ are representative images of PBS and PBA

nanofibers with around $70 \mathrm{~nm}$ diameter, respectively. As it can be seen, we have obtained nanofibers with good quality and high aspect ratio. 

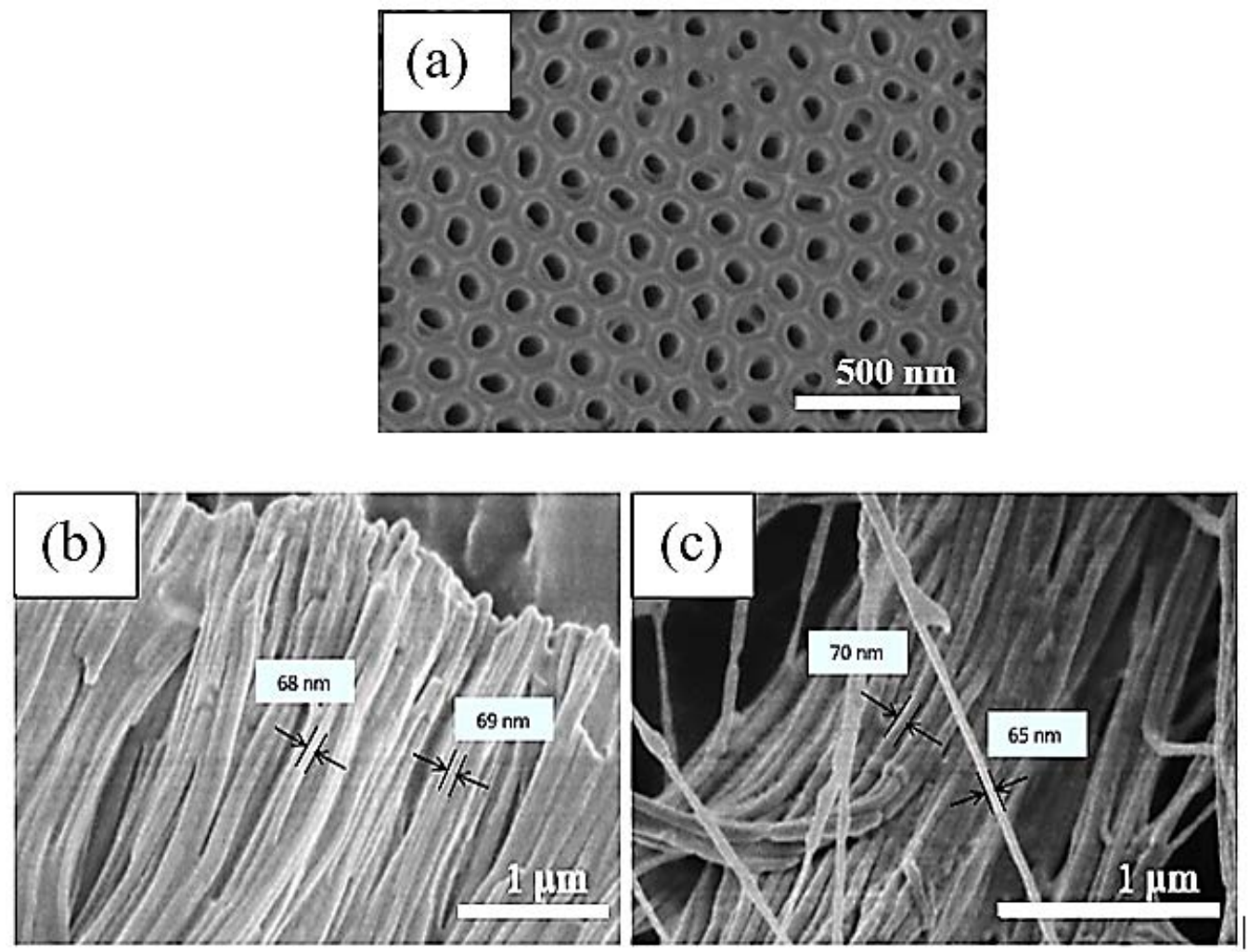

Figure 1. (a) SEM micrograph of the top view of the prepared AAO template with $70 \mathrm{~nm}$ pore size and $30 \mu \mathrm{m}$ length, (b) PBS and (c) PBA nanofibers produced inside the AAO templates (after the dissolution of the template in $1 \mathrm{M} \mathrm{NaOH}$ solution for $1 \mathrm{~h}$ ).

\section{Raman and FTIR spectroscopies}

AAO templates filled with PBS and PBA nanofibers were analyzed by Raman spectroscopy to corroborate that the infiltration of the polymers had been carried out successfully. PBS and PBA in bulk were also studied to compare their spectra with those of the infiltrated materials. For the characterization of Raman spectroscopy, first, the different main bands are assigned to each of the bonds that make up the PBS and PBA, respectively. The PBS and PBA spectra in bulk present less noise than confined samples and the bands are very well-defined, so these spectra will be used to compare with the spectra of infiltrated materials and to verify with their characteristic bands that the polymer has infiltrated correctly. 

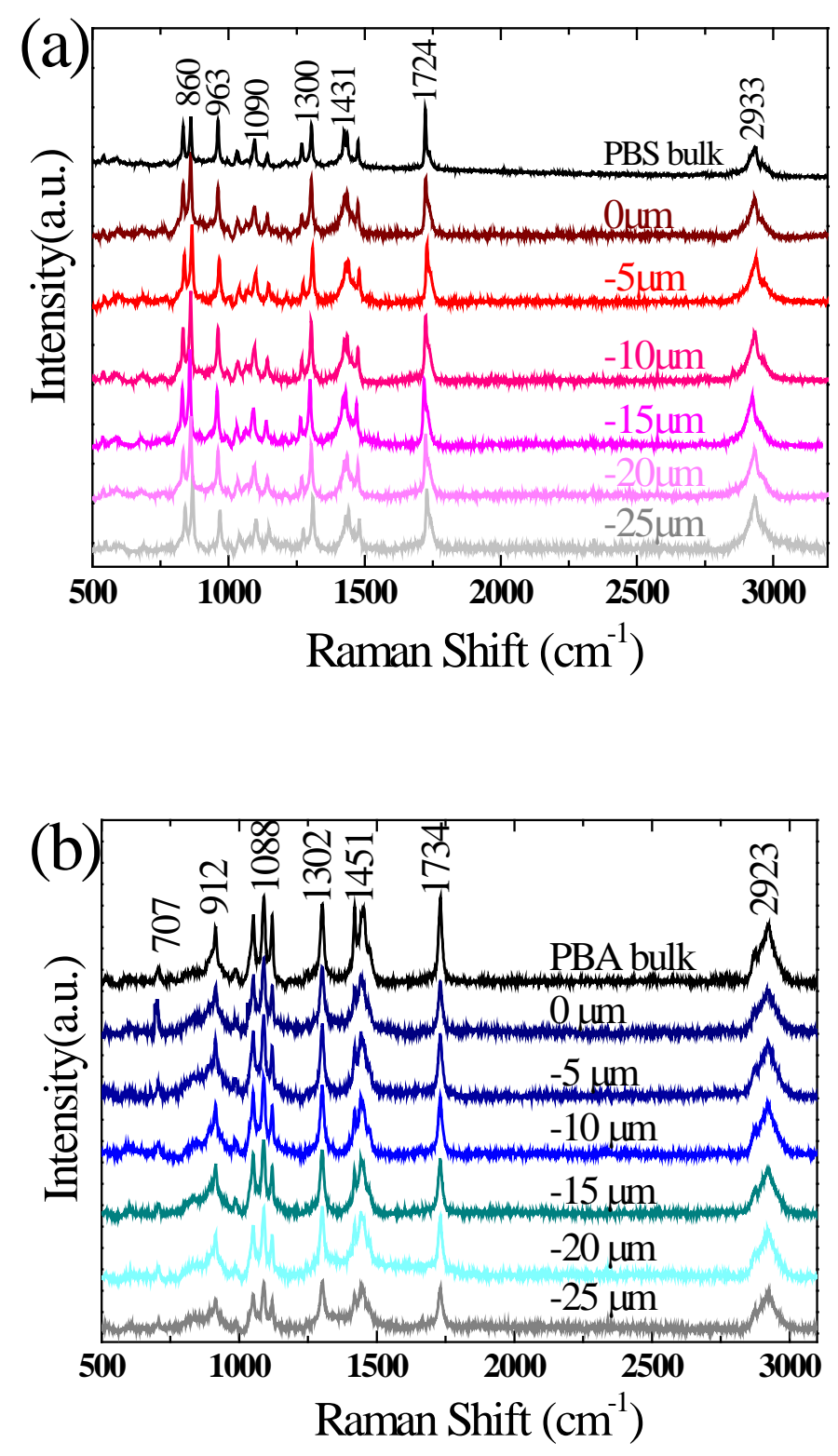

Figure 2. Depth profile Raman spectra of PBS (a) and PBA (b) in bulk, as references, and infiltrated within AAO templates with $70 \mathrm{~nm}$ pore size and $30 \mu \mathrm{m}$ length. Inserted numbers show the depth from the AAO surface.

For both polymers, the signals identified with specific bond vibrations according to the literature $\mathrm{H}^{40-42}$ are the following: around $2958 \mathrm{~cm}^{-1}$, asymmetric stretching mode of the $\mathrm{CH}_{2}$ groups; around $2875 \mathrm{~cm}^{-1}$, symmetric stretching mode of the $\mathrm{CH}_{2}$ groups; around $1721 \mathrm{~cm}^{-1}$, stretching 
mode of the $\mathrm{C}=\mathrm{O}$ bonds; around $1457 \mathrm{~cm}^{-1}$, bending in the plane of the $\mathrm{CH}_{2}$; and around 1121 $\mathrm{cm}^{-1}$, stretching mode of the C-O bonds.

Figure 2a and 2b show the normalized Raman spectra of infiltrated PBS and PBA inside AAO templates, with pinhole placement at different depths. Despite the noise due to the fluorescence of the alumina, it is possible to observe all characteristic bands of PBS and PBA up to $25 \mu \mathrm{m}$, respectively. As observed, all the bands remain constant along the depth of the template for both polymers, so, it confirms that both polymers penetrated homogeneously into the nanocavities.

Taking into consideration the SEM images of the nanofibers together with the Raman results and weight calculations of polymer infiltration, we can assume that the polymers were completely infiltrated into the full length of the nanopores $(30 \mu \mathrm{m})$.

Figure 3 shows FTIR spectra for PBS (a) and for PBA (b). Based on the literature ${ }^{42-47}$, characteristic absorption bands in the range $3100-2800 \mathrm{~cm}^{-1}$ are identified as $\mathrm{C}-\mathrm{H}$ vibrations of $\mathrm{CH}_{2}$ and $\mathrm{CH}_{3}$ groups. The strong bands at 1712 and $1725 \mathrm{~cm}^{-1}$ are due to the carbonyl group of the PBS and PBA ester groups, respectively. The other important bands are: $\mathrm{C}-\mathrm{H}$ bending at 1460 $\mathrm{cm}^{-1}$ and $\mathrm{C}=\mathrm{O}$ stretching at $1160-1170 \mathrm{~cm}^{-1}$. The signal at $1154 \mathrm{~cm}^{-1}$ is a characteristic of C-O$\mathrm{C}$ stretching vibration in the repeated $-\mathrm{OCH}_{2} \mathrm{CH}_{2}$ unit.

The most important differences in FTIR spectra between bulk and infiltrated samples are located at two regions that are labeled as $a$ and $b$ in Figure 3. In the dashed square labeled " $a$ " in Figure 3, i.e., region from $1100-850 \mathrm{~cm}^{-1}$, three strong absorption bands corresponding to the CO single bonds can be observed. For both polyesters, the band at $915 \mathrm{~cm}^{-1}$ (marked as dashed vertical line I) corresponds to the $\mathrm{C}-\mathrm{OH}$ bending vibration of the carboxylic acid groups and the 
band at $953 \mathrm{~cm}^{-1}$ (marked as dashed vertical line II) is due to the $\mathrm{O}-\mathrm{CH}_{3}$ stretching. Peaks at 1063 $\mathrm{cm}^{-1}$ for PBA and at $1045 \mathrm{~cm}^{-1}$ for PBS (marked as dashed vertical line III) correspond to $\mathrm{C}-\mathrm{C}-\mathrm{O}$ stretching vibration. The ratio of the intensity of the $\mathrm{C}-\mathrm{O}$ stretching and $\mathrm{C}-\mathrm{OH}$ bending peaks to the intensity of the $\mathrm{O}-\mathrm{CH}_{3}$ stretching peaks (III/II and I/II) increases in the nanofibers, as compared to the bulk. Also, the ratio of the intensity of the $\mathrm{C}-\mathrm{O}$ stretching band to the intensity of the $\mathrm{C}-\mathrm{OH}$ bending band (III/I) decreases in the nanofibers with respect to the bulk. These changes prove that nano-structuration affects the FTIR spectra. In fact, we can observe a smaller share of $\mathrm{O}-\mathrm{CH}_{3}$ bonds in the spectra, which could result from the interaction of PBS and PBA chains with the walls of the AAO templates. The differences between infiltrated and neat PBS spectra seem higher than those present in the case of neat and infiltrated PBA, but quantification of these signals is difficult.

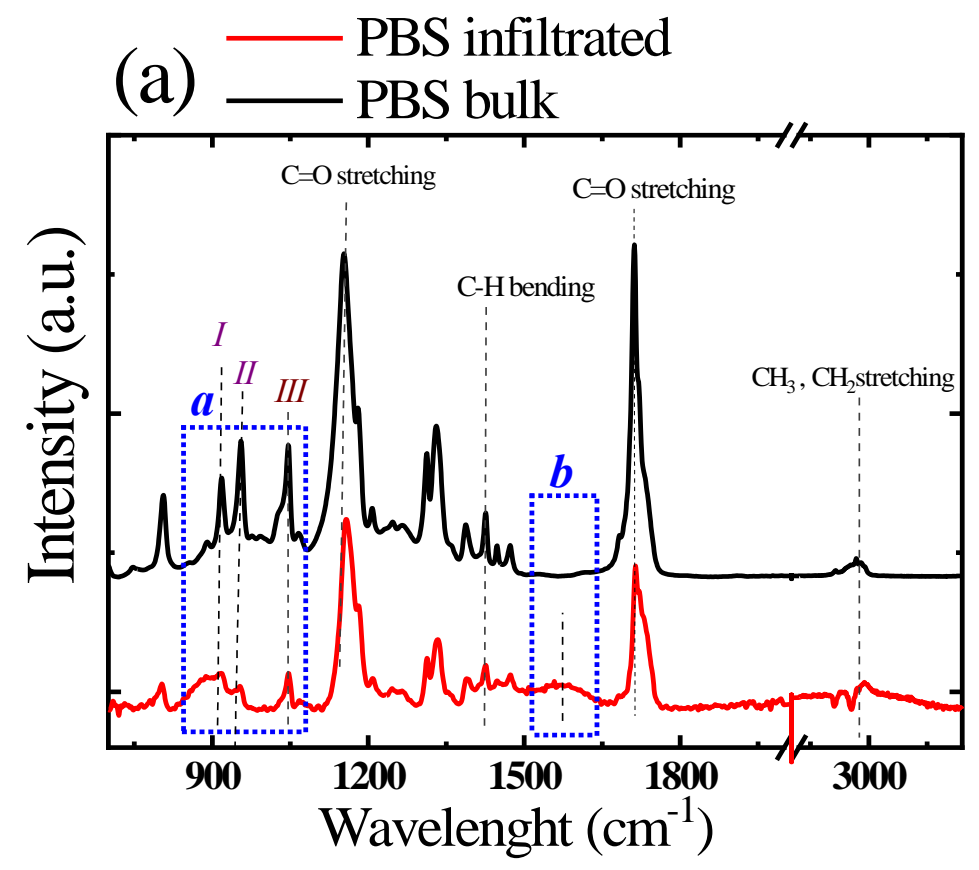




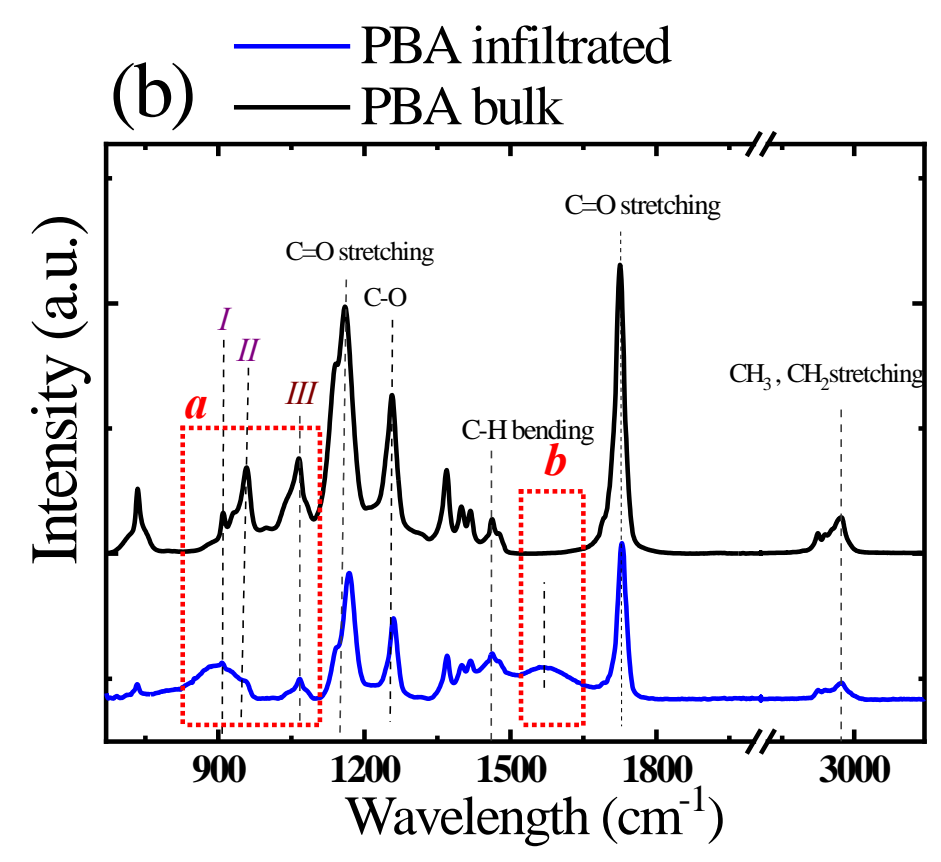

Figure 3. FT-IR spectra of PBS (a) and PBA (b) in bulk, as reference, and infiltrated in AAO templates with $70 \mathrm{~nm}$ nanopore size and $100 \mu \mathrm{m}$ length.

In addition, for both PBA and PBS, we observed a new intense band at $1571 \mathrm{~cm}^{-1}$ (see dashed square labeled "b” in Figure 3), which is characteristic of carboxylic acid salt stretching vibrations $\left(\mathrm{COO}^{-}\right)$, probably formed inside the AAO pores were small amounts of water moisture produced the hydrolysis of ester groups due to the hydrophilic character of the AAO template. ${ }^{48-}$ ${ }^{50}$ From the obtained results, it can be concluded that with nano-structuration of PBA and PBS, there is a decrease of interactions between the polymer chains and new interactions appear between the polymer chains and the walls of the AAO templates (i.e., $\mathrm{C}-\mathrm{O}$ on $\mathrm{O}-\mathrm{CH}_{3}$ ). Similar results were found for infiltrated PMMA as a function of the degree of confinement, by Raman spectroscopy. The Raman results helped to understand the effect of confinement on dielectric relaxation behavior of the PMMA. ${ }^{37}$ 


\section{Non-isothermal Crystallization}

DSC scans of PBS-bulk, PBA-bulk, PBS-nanofibers, and PBA-nanofibers inside $70 \mathrm{~nm}$ AAO templates are shown in Figure 4. The extracted data from DSC curves are summarized in Table 1. Figure 4a shows that during the cooling scan, PBA-bulk exhibits a crystallization peak at around $306 \mathrm{~K}$ that it is shifted drastically to $241 \mathrm{~K}$ when the PBA is confined within the alumina nanopores. The subsequent heating scans show that the melting point of confined PBA is slightly lower as compared to bulk PBA, $330 \mathrm{~K}$, and 326 K, respectively. Moreover, Figure 4b shows that the crystallization peak of PBS-bulk decreased from $355 \mathrm{~K}$ to $291 \mathrm{~K}$ in confined PBS and the peak melting temperature of PBS-bulk is $388 \mathrm{~K}$, whereas it is $385 \mathrm{~K}$ for infiltrated PBS.

Both infiltrated PBS and PBA show only a single crystallization exotherm at temperatures much lower than those in bulk (between 64 and $66 \mathrm{~K}$ lower respectively). The total absence of a crystallization peak at crystallization temperatures similar to those in bulk PBS or PBA indicates that the number of nanopores is much higher (possibly by several orders of magnitude) than the number of active heterogeneities capable of nucleating these polyesters in bulk at much lower supercooling. In other words, the nanopores are statistically clean, and they are also completely isolated and in no way interconnected. ${ }^{16,24}$ If after the infiltration process a polymer layer would have remained on top of AAO template, such layer could have interconnected pores and then spread heterogeneous nucleation by percolation. In this work, we have applied very careful cleaning procedures (see experimental section) to make sure that no remaining film is left after cleaning the surface of the AAO nanocavities following the recommendations of Shi et al. ${ }^{18}$, so heterogeneous nucleation can be ruled out. 

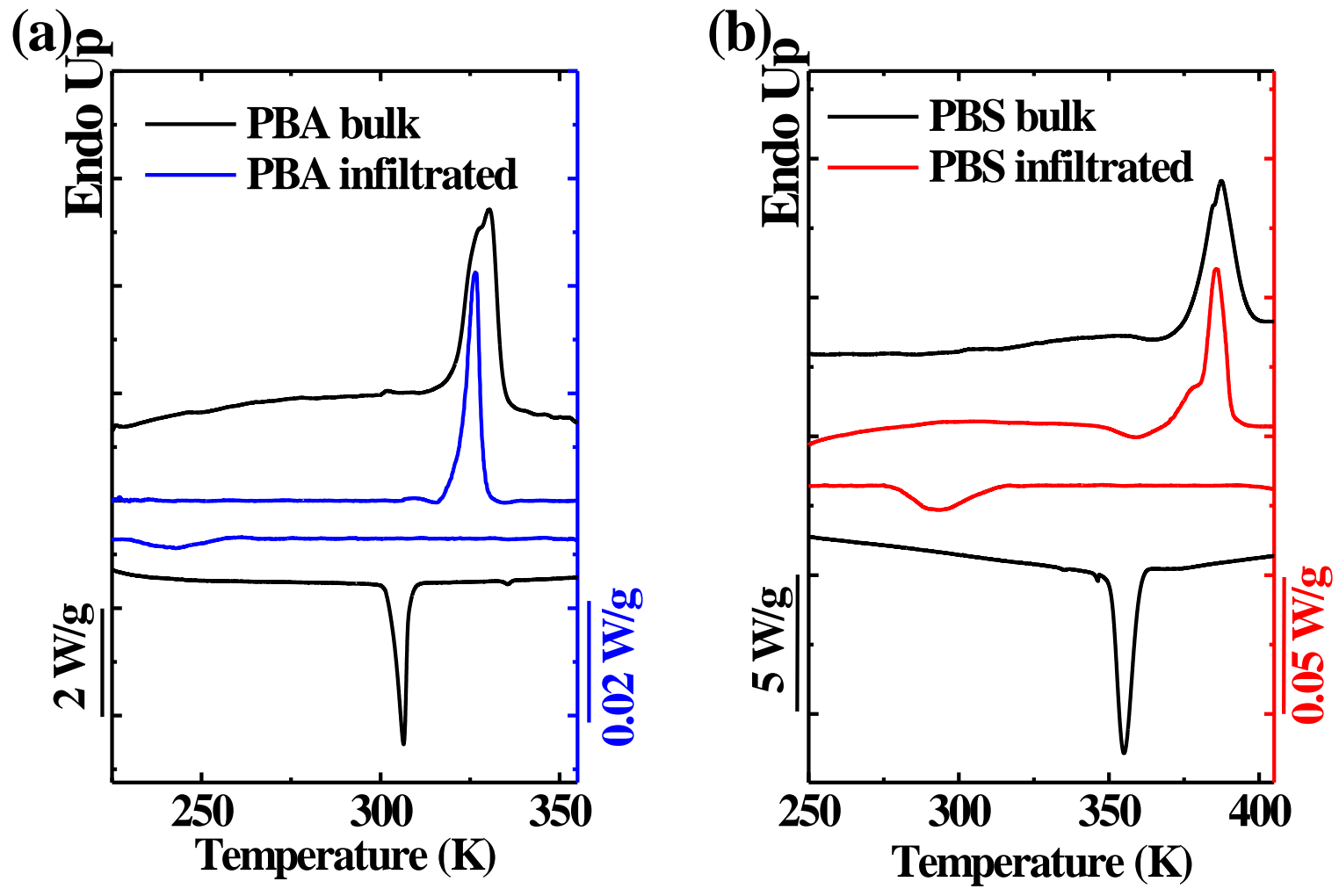

Figure 4. DSC curves for bulk and infiltrated samples of (a) PBA and (b) PBS.

Mi et al. ${ }^{51}$ studied how PBA nanotubes in alumina templates with 100 and $200 \mathrm{~nm}$ pore diameters crystallized, and they observed a very small shift of the non-isothermal crystallization temperature. This either indicates that all nanotubes contain similar heterogeneities as those of PBA in bulk, or that some PBA film remained on the surface of the AAO templates interconnecting the nanopores. However, our work is focused on how confinement affected phase transitions rather than in the nucleation of PBA. 
Table 1: Data Summary of DSC Results

\begin{tabular}{ccc}
\hline Sample & $\boldsymbol{T}_{\boldsymbol{m}}(\mathrm{K})$ & $\boldsymbol{T}_{\boldsymbol{c}}(\mathrm{K})$ \\
\hline PBS bulk & 388 & 355 \\
PBS Infiltrated & 385 & 291 \\
PBA bulk & 330 & 306 \\
PBA Infiltrated & 326 & 241 \\
\hline
\end{tabular}

The change in $T_{c}$ obtained upon infiltration in this work can be explained by a change in the nucleation mechanism of the polyesters, from heterogeneous to homogeneous or to surface nucleation. ${ }^{2}$ Infiltrated polymers can also nucleate at the AAO surface. ${ }^{29-30,52-53}$ It is difficult to distinguish between surface and homogeneous nucleation. ${ }^{28-30,54}$ However, if the crystallization temperature is very close to the glass transition temperature (5-15 K above the $T_{g}$ value), the nucleation will be most likely homogeneous. If on the other hand, the crystallization occurs at higher supercooling as compared to the bulk polymer, but still at considerably higher temperatures than $T_{g}$, then surface nucleation probably dominates the nucleation process within the nanopores. However, it must also be remembered that the homogeneous nucleation temperature is a function of the volume of the isolated nano or micro-domains. ${ }^{33}$

Comparing the $T_{g}$ value for PBA $(218 \mathrm{~K})$ with the crystallization temperatures reported in Table 1, we can deduce that the nucleation must have changed from heterogeneous for the bulk sample (with $T_{c}=306 \mathrm{~K}$ ) to homogeneous for the infiltrated sample (with a peak at $T_{c}=241 \mathrm{~K}$, notice that the exotherm is wide and ends at $228 \mathrm{~K}$ ), as the infiltrated sample is crystallizing at 
temperatures very close to the glass transition temperature. Therefore, in the infiltrated PBA case, the crystallization occurs just before vitrification in a single exotherm, a clear sign of confined crystallization initiated by homogeneous nucleation. 2, 29, 33, 54

The situation differs for the PBS case, as the crystallization changes from a peak value of $355 \mathrm{~K}$ to $291 \mathrm{~K}$ upon infiltration. Even though the change in supercooling is large, the $T_{g}$ of PBS is located at around $243 \mathrm{~K}$, hence still significantly much lower. Therefore, in the infiltrated PBS nanofibers, our DSC results indicate that the crystallization during cooling from the melt probably occurs by surface-induced nucleation caused by the AAO walls.

\section{Crystalline structure and chain orientation of PBS and PBA within nanopores}

To investigate the orientation and crystal texture of the PBA and PBS crystals within the AAO nanopores, 2D WAXS experiments with Grazing incident mode were carried out at room temperature.

Figure 5a and 5b show GIWAXS patterns for PBS-bulk and PBS-nanofibers (i.e., infiltrated PBS), respectively. There are five distinct reflections for PBS-bulk at $q=13.9,15.5$, 16.0, 18.4, $20.3 \mathrm{~nm}^{-1}$. These reflections have $d$-spacing values of 4.52, 3.97, 3.93, 3.41, and 3.09 $\AA$, which can be assigned to the following crystal planes: (020/111), (021), (110), (12 $\overline{1})$ and (111), respectively. Similar diffraction features can be found for the infiltrated PBS. Two major peaks with $q=13.8$ and $15.9 \mathrm{~nm}^{-1}$ can be indexed as the $(020 / \overline{1} 11)$ and $(021 / 110)$ reflections. Compared with the bulk PBS, the (021) and (110) reflections are not well resolved in the infiltrated sample. As shown in Figure S1 (see Supporting Information), the peak centered at 15.9 $\mathrm{nm}^{-1}$ can be fitted with two peaks. The small shoulder peak corresponds to the (021) reflection. 
Nevertheless, the difference is subtle, and we would not like to emphasize too much about the (021) peak. Therefore, we assign this peak to a combination of (021) and (110) reflections. The (111) reflection at $q=20.3 \mathrm{~nm}^{-1}$ is also visible in the infiltrated PBS. The results show that both the bulk and the infiltrated PBS crystallized into the same $\alpha$ form.
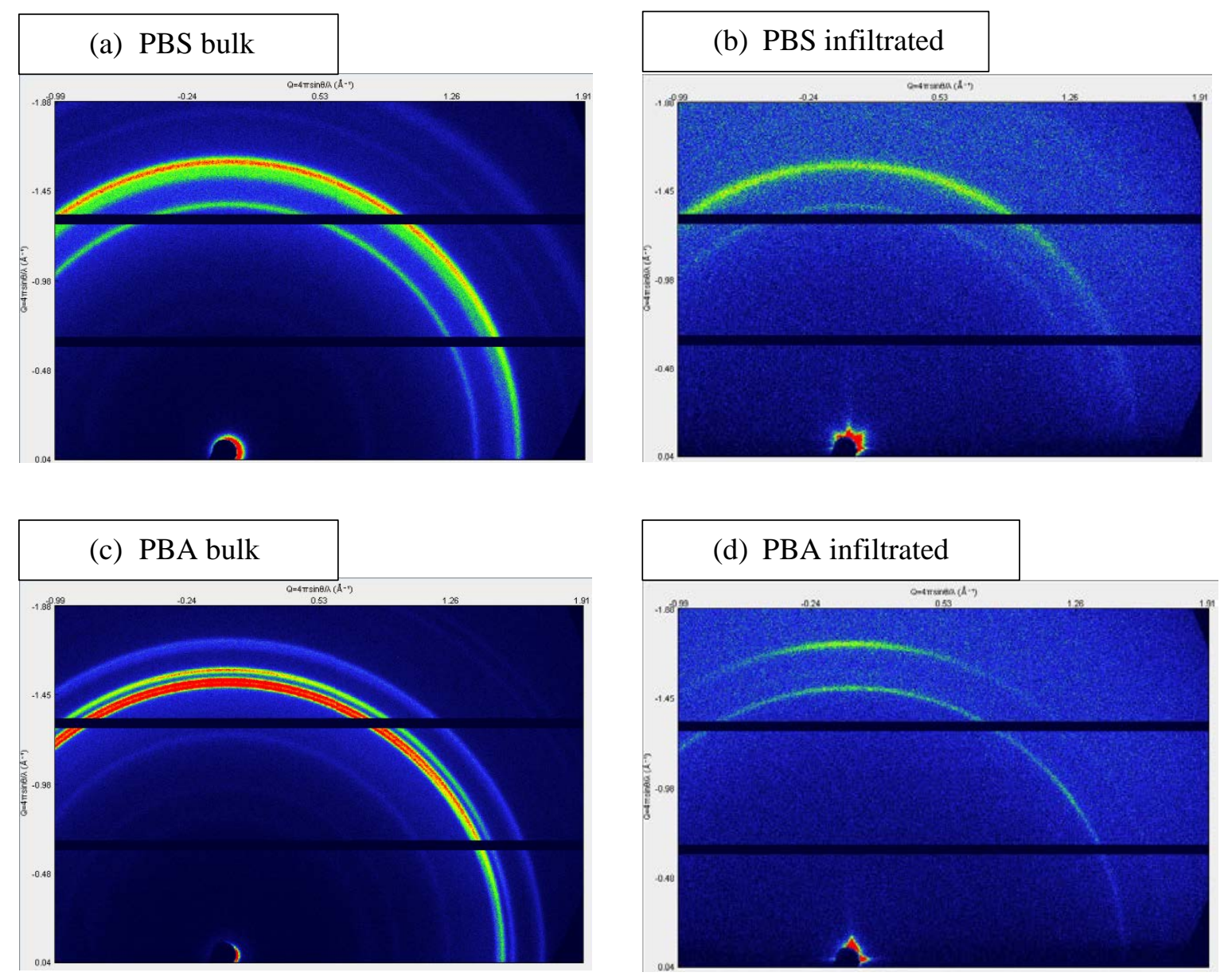

Figure 5. 2D WAXS patterns of (a) PBS-bulk (b) PBS infiltrated (c) PBA-bulk, and (d) PBAinfiltrated within AAO template with pore depth of $100 \mu \mathrm{m}$ and pore diameter of $70 \mathrm{~nm}$. X-ray incident angle with respect to the template is $3^{\circ}$.

Figure 5c and Figure 5d show the GIWAXS patterns of PBA-bulk and PBA-nanofibers, respectively. For PBA-bulk, main reflections were observed at $q=12.5,15.1,15.3,15.8,17.0$, and $17.2 \mathrm{~nm}^{-1}$. The reflections have $d$-spacing values of 5.03, 4.16, 4.10, 3.98, 3.69, and $3.65 \AA$, 
which can be assigned to the following crystallographic planes: $\alpha(002), \beta(110), \alpha(110), \alpha(020)$, $\alpha(021)$, and $\beta$ (020) reflections ${ }^{55-57}$, respectively. Hence, bulk PBA shows a mixture of two polymorphic phases, $\alpha$ and $\beta^{58}$ On the other hand, the reflection patterns of the PBA-nanofibers show only two $\beta$-crystals reflections at $q=15.1$ and $q=17.2 \mathrm{~nm}^{-1}$, which can be assigned to $\beta$ (110), $\beta$ (020) reflections. Sun et al. have reported similar results, as they only found $\beta$-crystals reflections, for PBA infiltrated within AAO templates. ${ }^{51,59}$

In both polymers, the scattered intensity inside $\mathrm{AAO}$ is poorer than the scattered intensity in bulk. This could be associated with the reduced amount of polymer inside the AAO template, in conjunction with a decrease in the degree of crystallinity.

Another feature of confined crystallization of polymers within AAO is the usual orientation of crystals inside the nanopores. As shown in Figure 6a, the degree of anisotropy of the PBS nanofibers is quite low as compared with other polymers such as PCL ${ }^{18}, \mathrm{PEO}^{17}$, and iPP. ${ }^{18}$ One possible reason is that the two major scattering arcs contain more than one reflection. If we assume the normal case of " $c$ axis perpendicular to the pore axis" ${ }^{21}$, the (020) and (110) should locate on the meridian, while the (111) and (021) should locate somewhere on the offmeridian position. Therefore, the mixed reflections could cause the broad azimuthal distribution of the two major arcs (see schematic illustration in Figure 6c). 

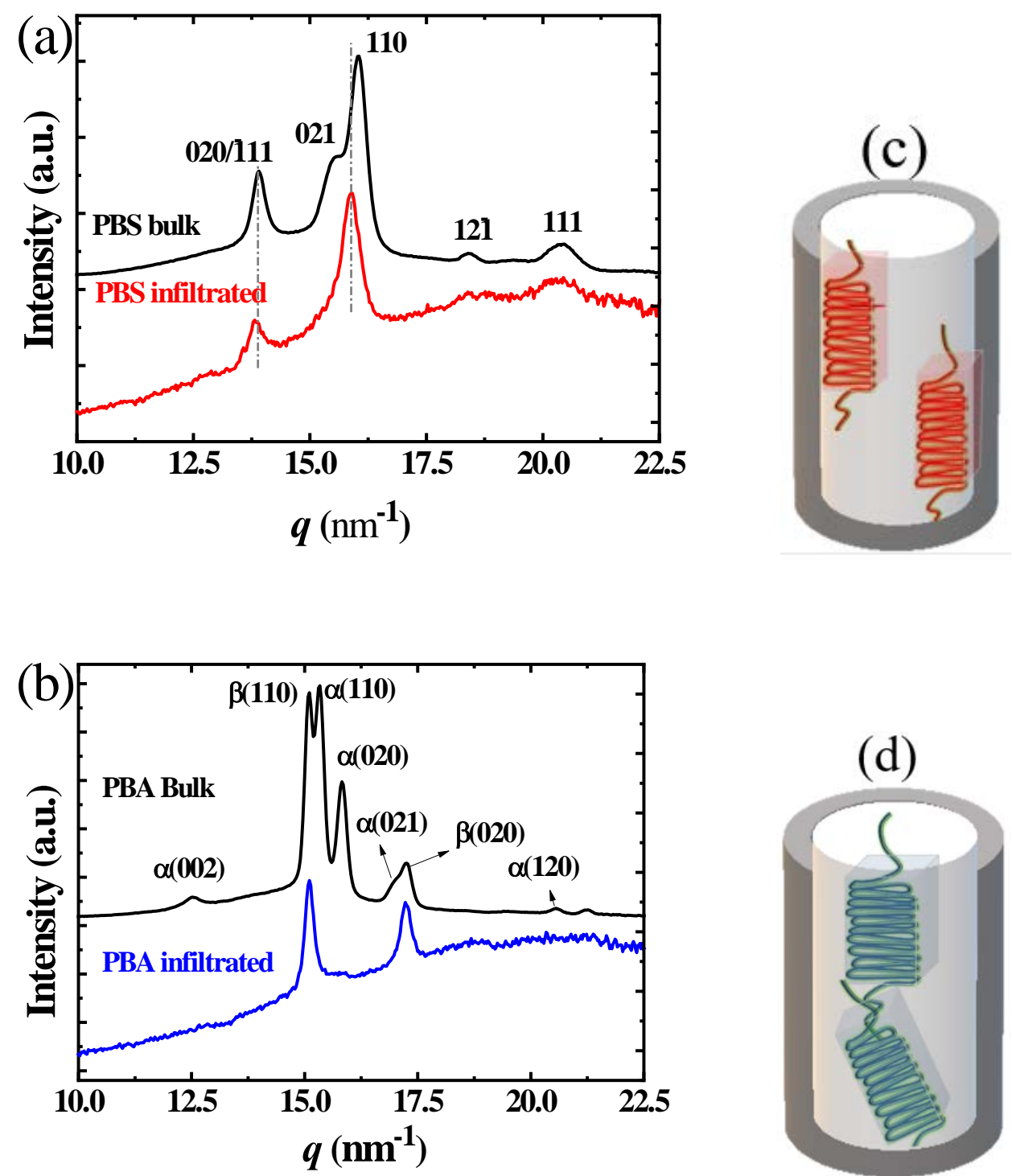

(d)

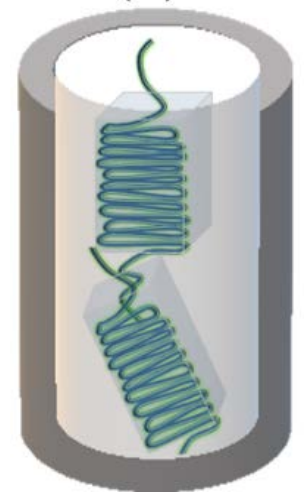

Figure 6. Corresponding 1D intensity profile of PBS (a) and PBA (b) samples, obtained by averaging the intensity along the azimuthal angle. Schematic illustration showing the cross section of a PBS (c) and PBA (d) nanofibers embedded in an AAO template with chain-folded lamellar crystals inside the nanopores.

On the other hand, infiltrated PBA exhibits a higher degree of anisotropy, as it is clear that the (020) reflection locates predominately on the meridian (see figure 6b). Azimuthal intensity profiles (see Figure 7) show that the intensity of the (020) peak is strongest on the meridian 
(azimuth $=90^{\circ}$ ). Two peaks are observed for the (110) reflection at azimuth $=90^{\circ}$ and $36^{\circ}$. The $\beta$ form of PBA has an orthorhombic unit cell with lattice parameters of $a=0.505 \mathrm{~nm}, b=0.736$ $\mathrm{nm}$, and $c=1.47 \mathrm{~nm}^{60-61}$, resembling crystalline structures of poly(tetramethylene adipate). ${ }^{62}$

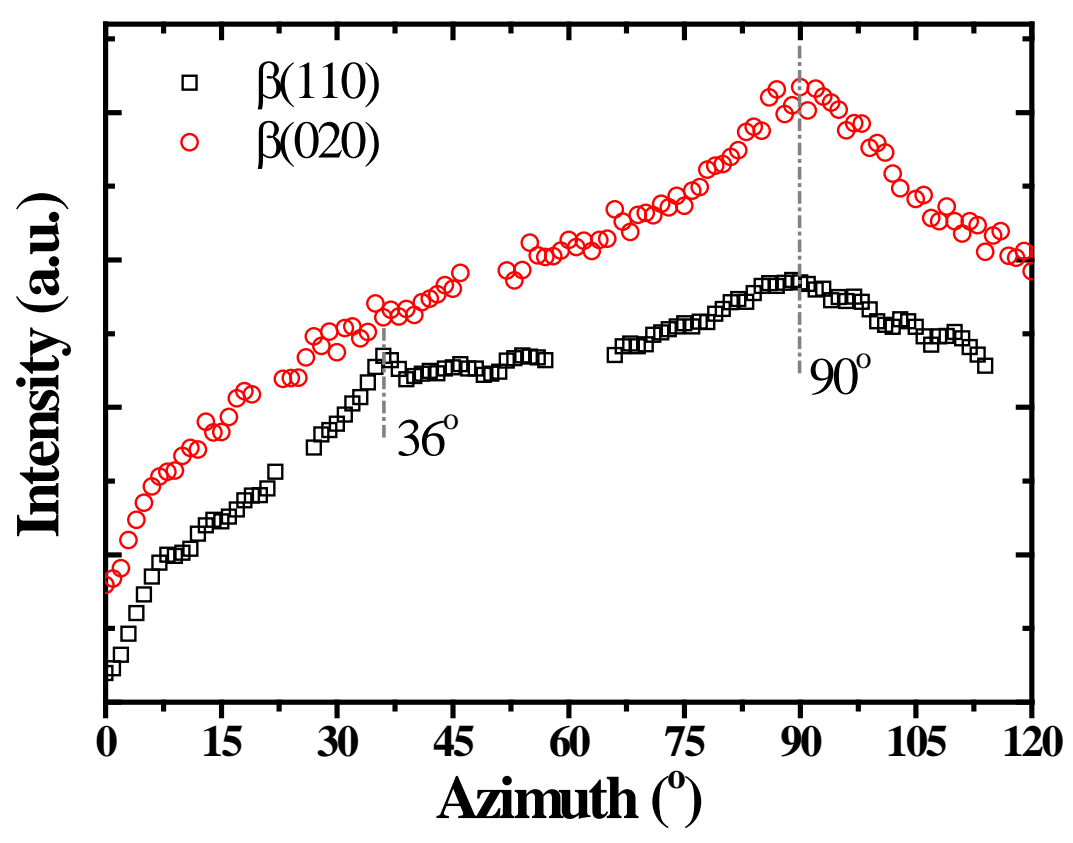

Figure 7. Azimuthal intensity profiles of the two major reflections of the infiltrated PBA sample.

The Polanyi equation is given by ${ }^{63}$ :

$$
\cos (\varphi)=\cos (\theta) \cos (\phi)
$$

where $\theta$ is the half Bragg angle and $\phi$ the azimuthal angle with respect to the meridian. Plugging $\theta=10.7^{\circ}$ and $\phi=54^{\circ}\left(90^{\circ}-36^{\circ}\right)$ into the equation yields a $\varphi$ value of $55^{\circ}$, which agrees with the angle between the (110) plane and the (020) plane for PBA infiltrated crystals. The meridional (020) and the (110) shoulder peak at azimuth $=36^{\circ}$ correspond to the orientation mode of $b$-axis parallel to the pore axis (see schematic illustration in Figure 6d). 
On the other hand, the (110) reflection for infiltrated PBA crystals has a broad peak at azimuth $=90^{\circ}$, which may indicate another possible orientation mode with the (110) plane normal parallel to the pore axis. We thus conclude that the PBA crystals within AAO templates exhibit a mixed orientation of $b$-axis or the (110) plane normal, parallel to the pore axis. Sun et al. reported an orientation mode of $b$-axis parallel to the pore axis for infiltrated PBA. ${ }^{51}$ However, the crystallization temperatures of their infiltrated PBA samples are 301 K (200 nm) and 299 K (100 $\mathrm{nm})$, much higher than that of our sample (241 K, $70 \mathrm{~nm})$.

Our recent study showed that the orientation of crystals within AAO is determined by the fastest growth direction of crystals. ${ }^{32}$ Therefore, the frequently observed mixed orientation in infiltrated polymers within AAO templates is rationalized by the comparable growth rates along several different crystal planes. This scenario works for PBA as well, because the (110) and (010) growth planes are the two fastest growth planes of PBA, as shown by the morphology of solution crystallized single crystals. ${ }^{64}$

\section{Broad Band Dielectric Spectroscopy for bulk and confined polymers.}

The dielectric loss, $\varepsilon^{\prime \prime}$, measured as a function of temperature is presented for bulk and infiltrated PBA and PBS samples in Figures 8a and 8b.

In both cases, two relaxation regions are observed: $\beta$ and $\alpha$ in the order of increasing temperature. The relaxation processes appear as a peak in the dielectric loss spectra, moving towards higher temperatures as frequency increases. Two frequencies were plotted for comparison purposes, $10^{1}$ and $10^{3} \mathrm{~Hz}$. At high temperatures, both polymers show the so-called $\alpha$ relaxation (or long-range segmental relaxation) which is the dielectric manifestation of the glass transition. In this relaxation process, the relaxation time strongly increases as temperature decreases. 

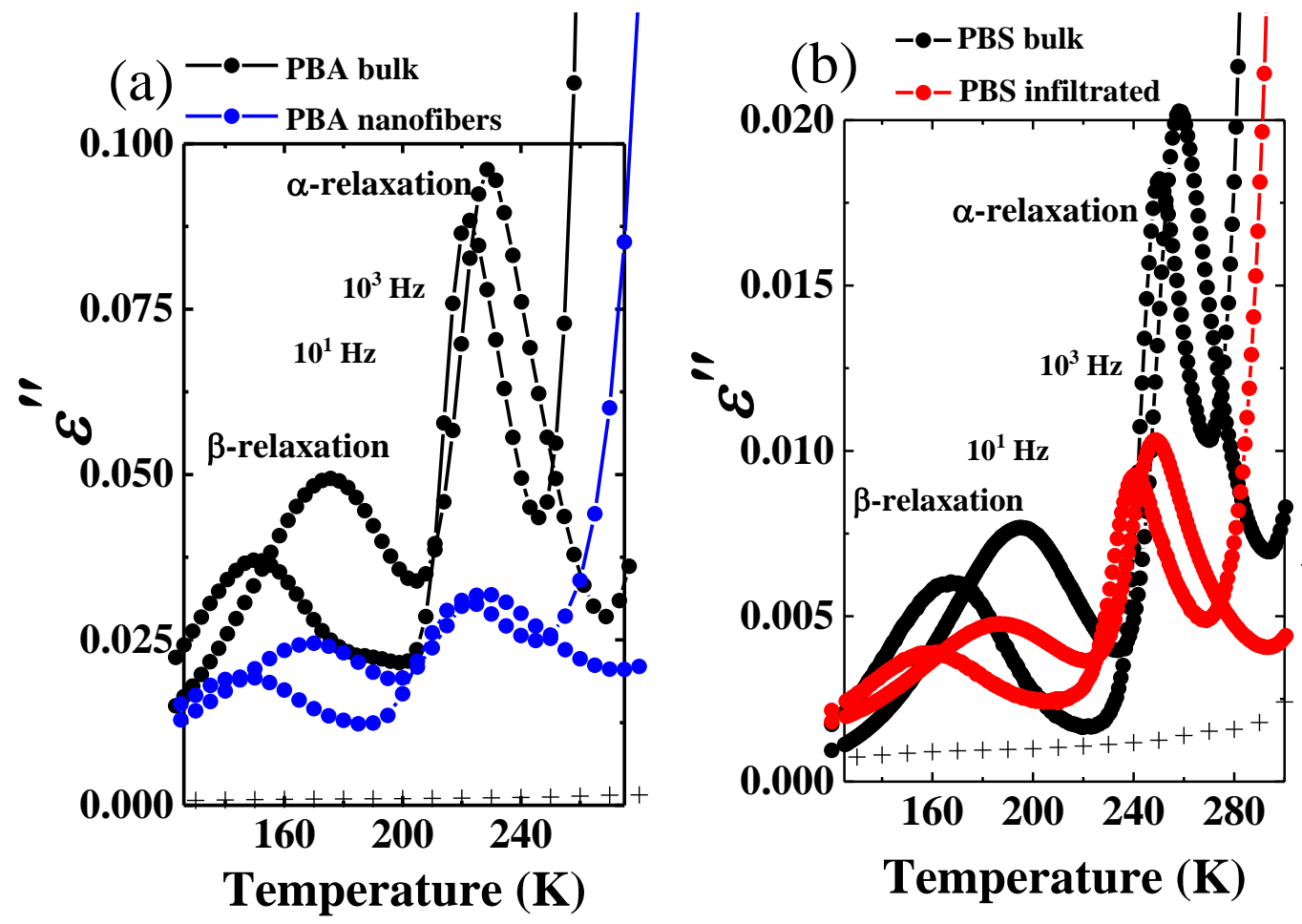

Figure 8. Isochronal plots of $\varepsilon^{\prime \prime}$ for (a) PBA bulk and PBA infiltrated in $70 \mathrm{~nm}$ alumina template, and (b) PBS bulk and PBS infiltrated in $70 \mathrm{~nm}$ alumina template at $10^{1}$ and $10^{3}$ $\mathrm{Hz}$. Loss curves of empty AAO templates $(+)$ at $10^{3} \mathrm{~Hz}$ are included in both Figures for comparison purposes.

A secondary $\beta$-relaxation is also detected in Figure 8 . The $\beta$ relaxation is due to the shortrange motions of lateral or short-chain segments, which occur below the glass transition temperature. ${ }^{65}$ For this kind of semicrystalline polyesters, where the dielectric relaxations are related with reorientations of the dipole moments in the ester groups, a single $\beta$-relaxation component is observed, as Arandia et al. recently reported. ${ }^{66}$ To get this single $\beta$-relaxation peak, the samples have to be carefully dried before the dielectric experiments are performed. ${ }^{67}$

Figure 8 also shows data for PBA and PBS infiltrated within $70 \mathrm{~nm}$ pore AAO templates. For these samples, the dielectric losses have a lower contribution than the bulk samples (due to the reduction of the volume of the sample capacitor filled by the polymer) and an additional 
background-like signal from the alumina template that is weakly dependent on frequency and temperature. Relaxation processes (i.e., $\alpha$ and $\beta$ ) move to lower temperatures at given frequencies when the polymers are confined within the alumina nanopores.

Representative isothermal experiments in the temperature range relevant for the $\beta$ relaxation were carried out to analyze in detail the changes observed. The isothermal permittivity loss versus frequency for all samples at different temperatures is presented in Figure 9. In this figure, the main peaks observed correspond to the $\beta$-relaxation, and it is possible to compare quantitatively the differences in relaxation rates between bulk polymers and infiltrated samples better than in the isochronal plots case (Figure 9).
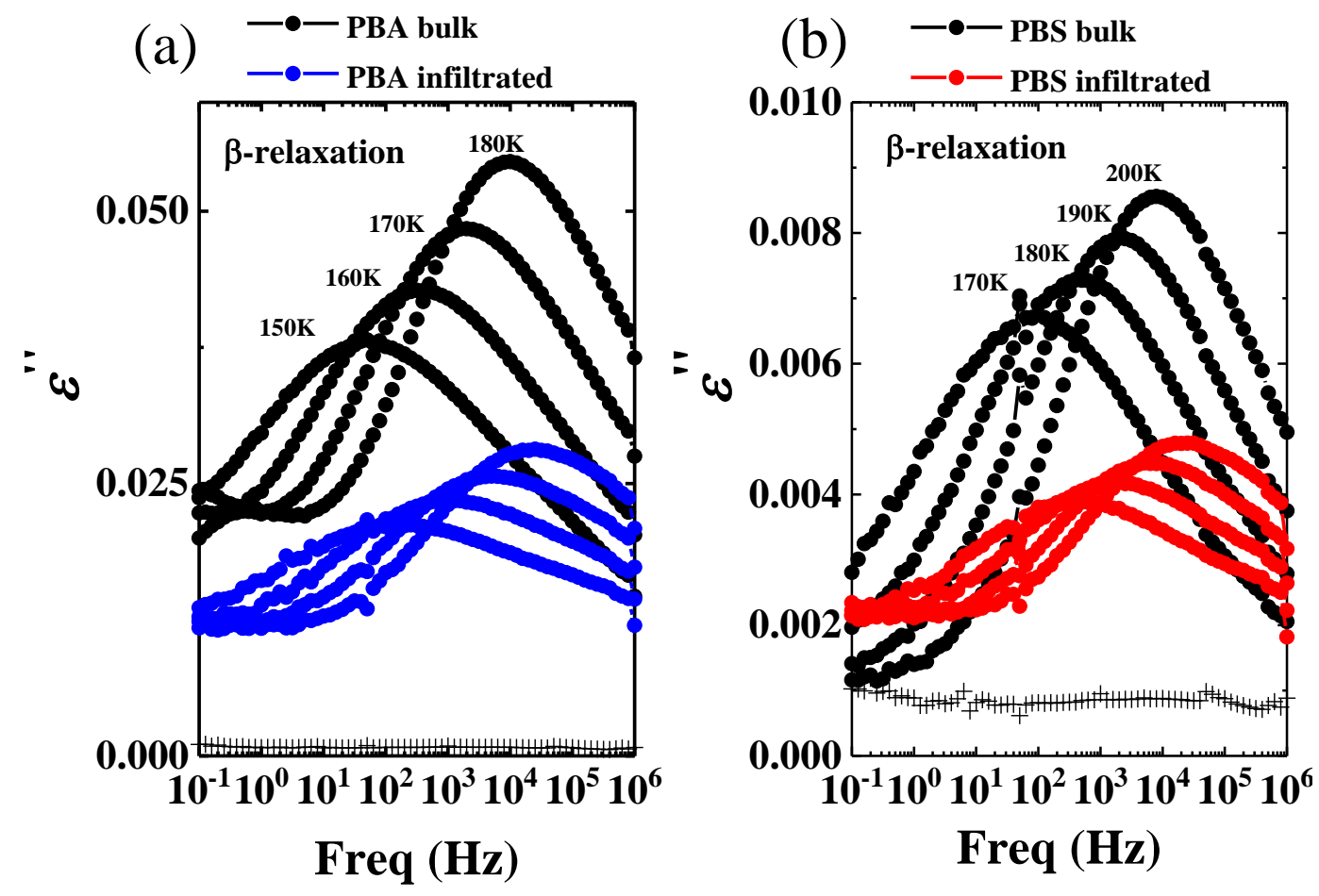

Figure 9. Isothermal plots of $\varepsilon^{\prime \prime}$ for (a) PBA bulk and PBA infiltrated in $70 \mathrm{~nm}$ alumina template at 150,160, 170 and 180K, and (b) PBS bulk and PBS infiltrated in $70 \mathrm{~nm}$ alumina template at 170, 180, 190 and $200 \mathrm{~K}$. Loss curves of empty AAO templates (+) at $160 \mathrm{~K}$ for Figure 9a and at 180K for Figure 9b are included for comparison purposes. 
In the $\beta$-relaxation region, the peak frequency, $f_{\max }$, of confined polymers occurs at higher frequencies than those of bulk samples, indicating a faster local dynamics (see Supporting Information Figure S2). Some conformational changes also explained and observed above by FTIR can cause these faster dynamics in the $\beta$-relaxation when the samples are infiltrated into alumina nanopores. Confined effects can be due to the limited small spaces within the alumina nanocavities or to surface nucleated alumina walls. To quantify these differences, the relaxation time from each isothermal plot has been calculated as:

$$
\tau=\left(2 \pi f_{\max }\right)^{-1}
$$

For both systems, a clear Arrhenius behavior is found, and consequently, the data have been fitted to the Arrhenius equation:

$$
\tau(T)=\tau_{\infty} \exp \left[\frac{E_{a}}{k_{B} T}\right]
$$

The resulting values are presented in Table 2. The activation energies for both bulk samples, PBA and PBS, are about 10\% higher than those obtained for infiltrated samples. 
Table 2. Parameters describing the temperature dependence of the $\beta$-relaxation and $\alpha$-relaxation.

\begin{tabular}{|c|c|c|c|c|c|c|c|c|}
\hline \multirow[b]{2}{*}{ Sample } & \multicolumn{4}{|c|}{$\beta$-relaxation } & \multicolumn{4}{|c|}{$\alpha$-relaxation } \\
\hline & $\begin{array}{c}E_{a} \\
(\mathrm{~kJ} / \mathrm{mol})\end{array}$ & $\begin{array}{l}\tau_{\infty} \\
(s)\end{array}$ & $\begin{array}{c}\Delta H \\
(\mathrm{~kJ} / \mathrm{mol})\end{array}$ & $\begin{array}{c}\Delta S \\
(\mathrm{~kJ} \mathrm{~K} / \mathrm{mol})\end{array}$ & $\begin{array}{c}\mathbf{B} \\
(\mathrm{K})\end{array}$ & $\begin{array}{r}T_{0} \\
(\mathrm{~K})\end{array}$ & $\begin{array}{c}\mathbf{F}_{\infty} \\
(\mathbf{H z})\end{array}$ & $\begin{array}{l}T_{g} * \\
(\mathrm{~K})\end{array}$ \\
\hline PBA bulk & 41 & $1.7 \times 10^{-17}$ & 40 & 0.072 & 1186 & 183 & $1 \times 10^{14}$ & 214 \\
\hline PBA Infiltrated & 37 & $1.0^{\times} 10^{-16}$ & 6 & 0.058 & 1018 & 185 & $1 \times 10^{14}$ & 215 \\
\hline PBS bulk & 43 & $1.4^{\times} 10^{-16}$ & 41 & 0.054 & 1393 & 204 & $1 \times 10^{14}$ & 238 \\
\hline PBS Infiltrated & 38 & $4.3^{\times} \times 10^{-16}$ & 37 & 0.045 & 1387 & 195 & $1 \times 10^{14}$ & 229 \\
\hline
\end{tabular}

* $T_{g}$ value for the bulk is taken equal to that one obtained from DSC data. The extrapolation to the infiltrated one is taken when $\log \mathrm{f}(\mathrm{Hz})=\mathrm{a})-2.4$ for PBA and b) -3.5 for PBS from Figure 11.

The extremely low values obtained for $\tau_{\infty}$ indicate that the molecular origin of the $\beta$ relaxation does not correspond to single activated jumps between two equivalent positions of molecular units over an energy barrier, and therefore a more complex situation should be considered. One way of describing such behavior is by using the Eyring equation, where the relaxation time is expressed in terms of the difference in the Gibbs free energy, $\Delta \mathrm{G}=\Delta \mathrm{H}-\mathrm{T} \Delta \mathrm{S}$, between the equilibrium and the activated state. This leads to an equation similar to the Arrhenius one, where an entropic term appears in addition to the enthalpic one, as follows:

$$
\tau(T)=\frac{h}{k_{B} T} \exp \left[\frac{\Delta G}{k_{B} T}\right]=\frac{h}{k_{B} T} \exp \left[-\frac{\Delta S}{k_{B}}\right] \exp \left[\frac{\Delta H}{k_{B} T}\right]
$$

where $h$ is Planck's constant and $\Delta H$ and $\Delta S$ are the enthalpic and entropic changes respectively corresponding to the activated state. The same data are plotted in Figure 10 but with a linear behavior corresponding to equation 4. The extracted parameters are shown in Table 2. 

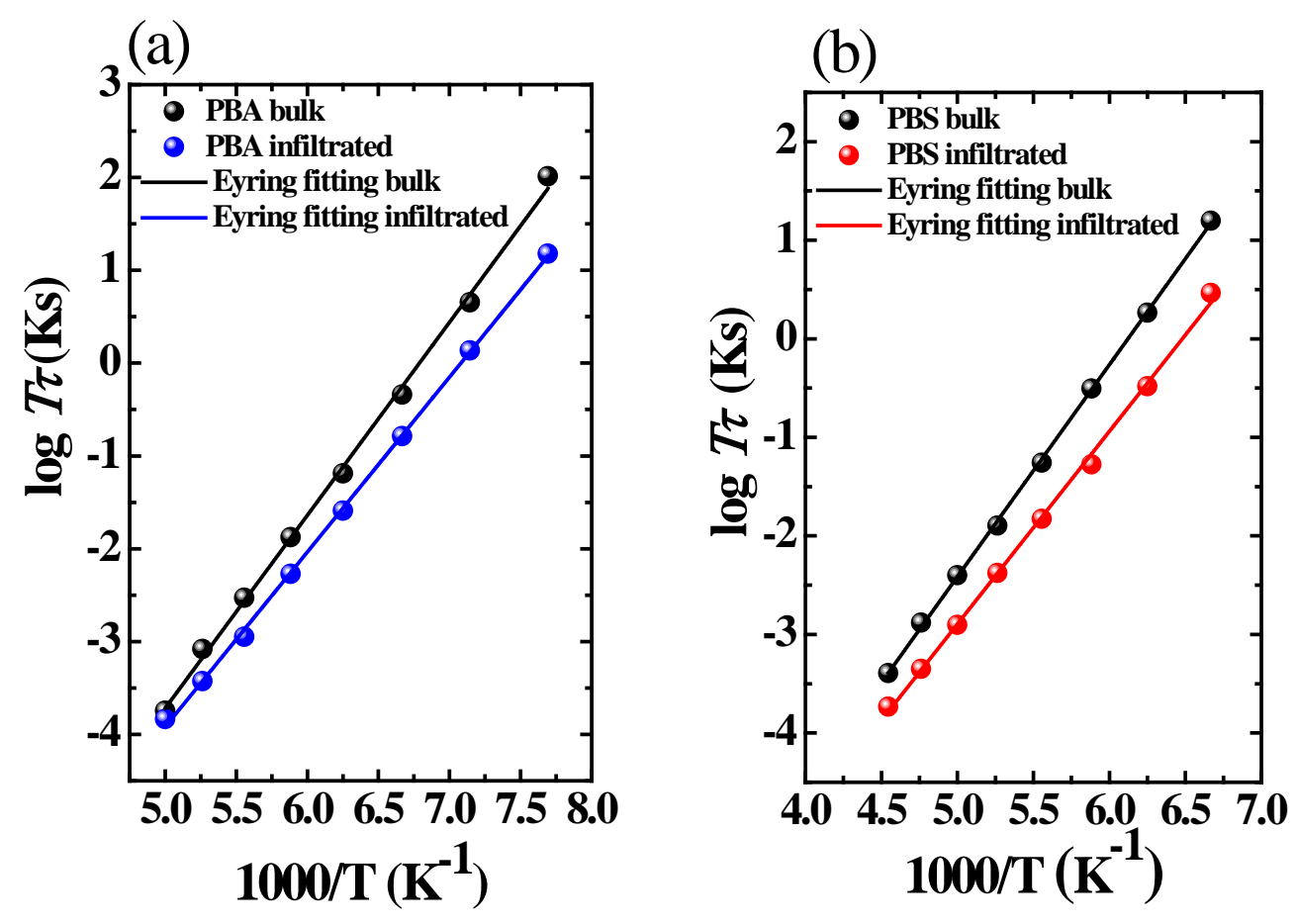

Figure 10. Arrhenius plots of $\beta$ processes where solid lines represent the Eyring descriptions for (a) PBA bulk and infiltrated PBA, and (b) PBS bulk and infiltrated PBS.

Both enthalpic and entropic terms observed for both bulk PBA and PBS take relatively large values as compared with the infiltrated samples. Accordingly, it seems that confinement in the nanopores not only reduce the energetic barriers but also made the molecular mechanism for reorientation less complex (lower activation entropy).

Concerning the $\alpha$-relaxation, it can be seen in Figure 6 that it is sharp and stronger as compared to the corresponding $\beta$-relaxation. The quantification of the $\alpha$-relaxation has been made by using the isochronal representation shown in Figure 11, since the conductivity contributions difficult a reasonable analysis of the isothermal curves from the infiltrated polymers, mainly in the case of PBA. 

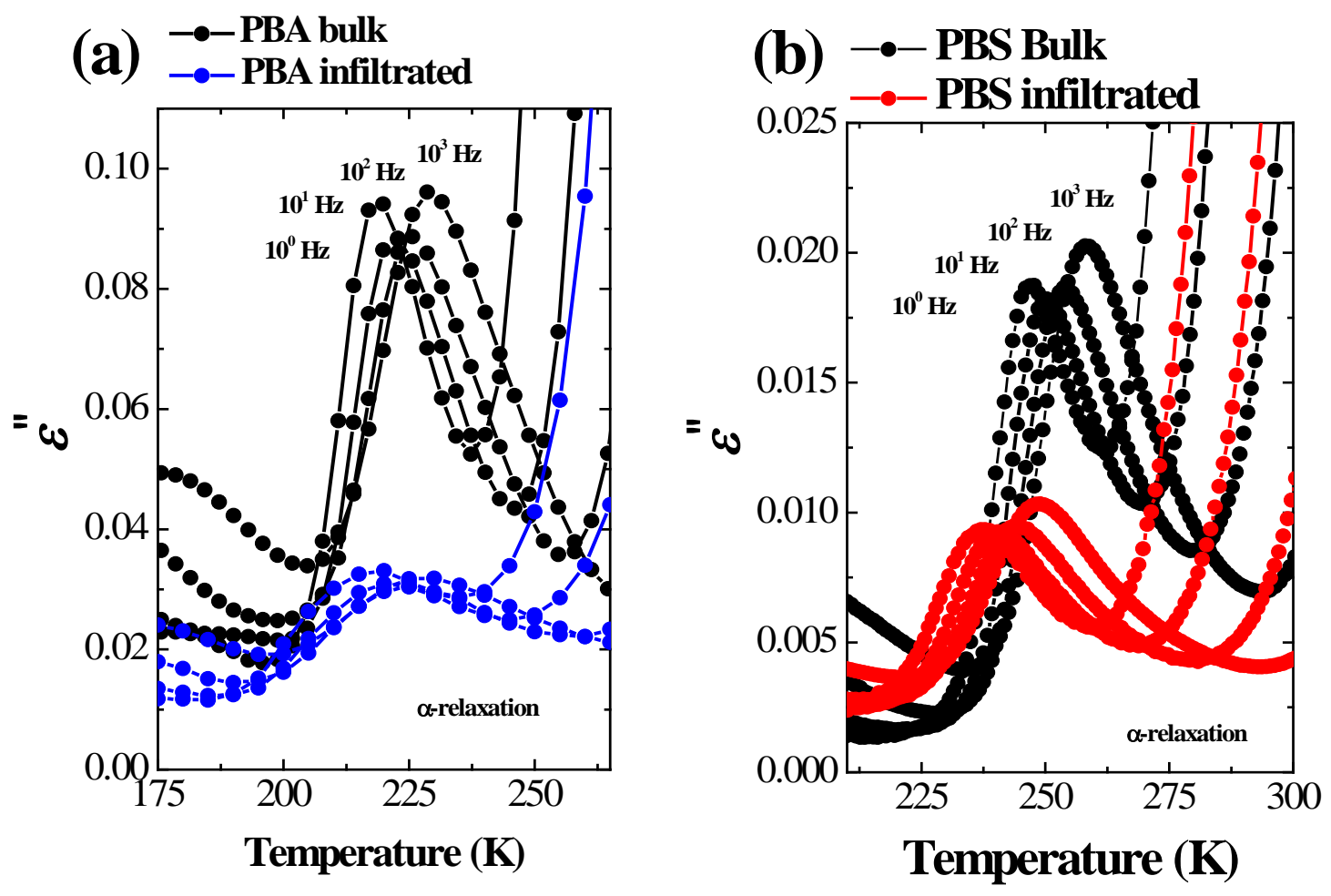

Figure 11. Isochronal plots of $\varepsilon^{\prime \prime}$ (a) PBA bulk and infiltrated PBA, and (b) PBS bulk and infiltrated PBS for $10^{0}, 10^{1}, 10^{2}$ and $10^{3} \mathrm{~Hz}$.

Using the isochronal representation of the dielectric losses at different frequencies, the temperature $\left(T_{\max }\right)$ at which the $\alpha$-relaxation loss peak occurs has been determined for all samples. The temperature dependence of the peak frequencies corresponding to bulk and infiltrated samples are shown in Figure 12. 

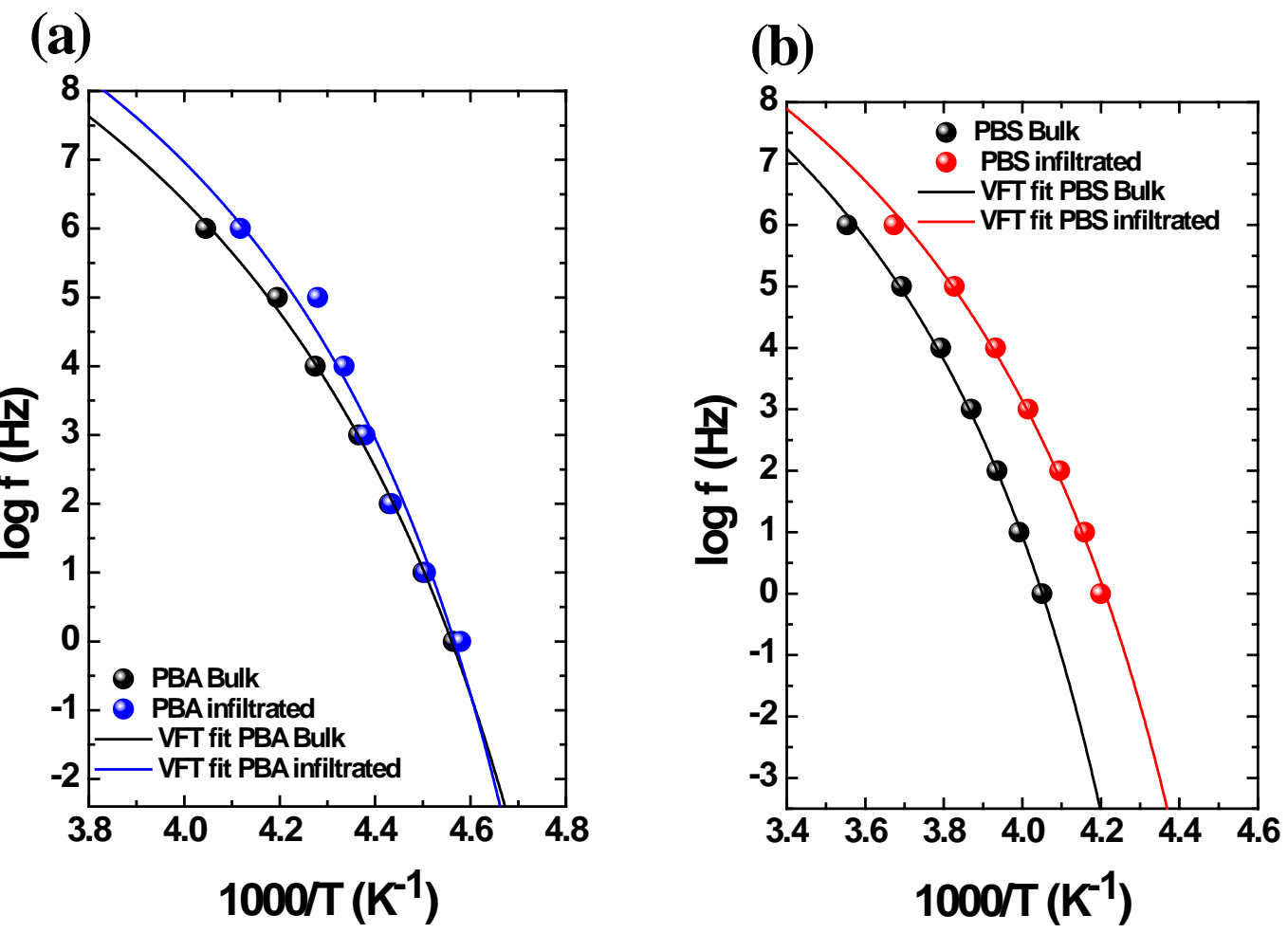

Figure 12. Arrhenius plot of the peak frequencies of (a) PBA bulk and infiltrated PBA, and (b) PBS bulk and infiltrated PBS. Solid lines represent the corresponding VFT fit of $\alpha$ relaxation.

The data in Figure 12 were fitted to a usual Vogel-Fulcher-Tamman (VFT) equation:

$$
f\left(T_{\max }\right)=f_{\infty} \exp \left(\frac{-B}{T_{\max }-T_{0}}\right)
$$

where $T_{0}$ is the Vogel temperature, $\mathrm{B}$ is an energetic term, and $f_{\infty}$ would correspond to a typical vibration frequency. The obtained fitting lines are shown in Figure 12, and the parameters are summarized in Table 2. To obtain accurate fits, and in accordance with the DSC $T_{g}$ values obtained for the bulk polymers, a value of $f_{\infty}$ of $1 \times 10^{6} \mathrm{~Hz}$ was assumed. 
The glass transition temperature obtained from the VFT fits is affected by confinement effects for both PBA and PBS samples. This glass transition temperature differences could be explained by taking into account the differences in the crystallinity between bulk and confined samples. However, the differences observed in $\alpha$-relaxation peaks are also in accordance with the differences observed before in the $\beta$-relaxation peaks. Smaller differences are observed for the PBA than for the PBS polymer. However, there are also differences in the curvature of the VFT lines. In this case, the curvature increases for PBA but decreases for PBS. The behavior of PBA is quite unusual and could be due to a combination of two effects, one associated with faster local dynamics and the other hindering the cooperative motions involved in the glass transition. Consequently, it seems that these relaxations are quite dependent on the nature of the material used.

DSC experiments have also detected important differences in the crystallization of confined PBA and PBS, as in PBA the nucleation is probably homogeneous, but in the case of PBS, it is induced by the AAO walls (i.e., surface nucleation). Moreover, it is worth considering that confinement and surface effects of the nano-constraint polymers may not necessarily be identical over the entire frequency or temperature range of the relaxation response.

The results compiled in Table 2 suggest some correlation between the $\alpha$-relaxation and the $\beta$-relaxation, although both processes are located at different frequencies and temperature ranges. The different values obtained for $\mathrm{E}_{\mathrm{a}}$ ( $\beta$-relaxation) and $T_{0}$ ( $\alpha$-relaxation) reported for the confined samples in this manuscript, confirm that confinement effects are playing a large role in these samples, and that is why the dynamics of the PBA and PBS chains are different from those obtained for the bulk samples.

Furthermore, there are large differences in the trends experienced by the glass transition temperature (see Table 2) upon confinement. PBA either experiences a very slight increase in $T_{g}$ 
upon infiltration (i.e., $1 \mathrm{~K}$ ) or does not change with respect to the bulk if we consider the difference not significant. On the other hand, the $T_{g}$ of PBS decreases $9 \mathrm{~K}$ with confinement (see Table 2). These differences may be correlated with the different interactions that the polymer chains have with the AAO walls in addition to confinement effects.

In several infiltrated polymers, the detection of two $T_{g}$ transitions (or even $3 T_{g}$ transitions) has been reported. This prompted researchers to interpret these two $T_{g}$ values, as arising from two different layers within the infiltrated material. Typically, one $T_{g}$ is much lower than the bulk $T_{g}$ value and the other one, either stays at temperatures close to the bulk value or is even higher. If we consider the pores as perfect cylinders, we can divide each pore into two concentric cylinders. One of them consists of chains with higher segmental long-range mobility in the amorphous regions (the part with the lower $T_{g}$ values), while the other has chains with more restricted mobility, comparable or even slower than those in the bulk material (the part with the higher $T_{g}$ values). ${ }^{68-71-72}$

In our case, only one $T_{g}$ relaxation process has been detected for both PBS and PBA. However, we speculate that in the PBS case, where the material is more likely to nucleate at the AAO walls, a rigid semi-crystalline layer could be produced around the pore walls that do not contribute to the $\alpha$-process that we detect by dielectric experiments or DSC (maybe because the layer is thin and its mobility is compromised by the crystals present and by the interactions with the walls). Instead, the dominant mobile amorphous chains present in the core experience higher segmental mobility and concomitantly a lower $T_{g}$ value.

In the homogenously nucleated PBA case, the nucleation should occur within the volume of each nanopore (a characteristic of homogenous nucleation, see refs. 2-3) and away from the interface with the AAO walls. As the $T_{g}$ is similar than that of the bulk, we speculate that 
segmental mobility is in this case dominated by confinement within the pores and is less affected by the presence of the AAO walls. ${ }^{23}$

\section{CONCLUSIONS}

Polybutylene succinate (PBS) and Polybutylene adipate (PBA) were successfully infiltrated within laboratory-made Anodic Aluminum Oxide (AAO) templates of $70 \mathrm{~nm}$ diameter. DSC results indicate that infiltrated PBA molecules nucleate homogeneously inside the pore volume while BDS measurements showed that $T_{g}$ values did not significantly change upon infiltration. On the other hand, PBS probably nucleates at the surface of AAO walls and the possible interactions with alumina were detected by FTIR. Such interaction with AAO walls influenced chain dynamics in such a way that BDS studies found a depression of approximately $9 \mathrm{~K}$ in the glass transition temperature upon infiltration. We conclude that PBS displays a higher affinity with AAO walls in comparison with PBA. Some differences were also found in terms of polymorphic behavior and crystal orientation between PBS and PBA by GIWAXS. Our study shows that the consequences of confinement in $70 \mathrm{~nm}$ diameter AAO templates are clearly different for PBA and PBS.

\section{ACKNOWLEDGMENTS}

POLYMAT and ICTP-CSIC Institutions acknowledge financial support from the Spanish Ministry of Science, Innovation, and Universities (MAT2017-83014-C2-1-P and MAT201783014-C2-2-P). M.S. gratefully acknowledges the award of a Ph.D. fellowship by POLYMAT Basque Center for Macromolecular Design and Engineering. J.M. acknowledges support from the Provincial Council of Gipuzkoa under the program Fellow Gipuzkoa and "Fomento San Sebastián” in the framework program “Retorno del Talento Local” Donostia up! 2016. G.L, D.W., and A.J.M acknowledge the support from the National Natural Science Foundation of China 
(51820105005, 21873109) and National Key R\&D Program of China (2017YFE0117800). G.L. acknowledges the support from the Youth Innovation Promotion Association of the Chinese Academy of Sciences (2015026). This work has received funding from the European Union's Horizon 2020 research and innovation program under the Marie Sklodowska-Curie grant agreement No 778092. The UPV/EHU team gratefully acknowledges the financial contribution of the Basque Government through grant IT1309-19.

Supporting Information:

Figure S1: 1D intensity profile of PBS infiltrated sample

Figure S2: Isothermal plots of $\varepsilon^{\prime \prime}$ for a) PBA homopolymer and PBA infiltrated

\section{REFERENCES}

1. Fujimaki, T., Processability and properties of aliphatic polyesters, 'BIONOLLE', synthesized by polycondensation reaction. Polymer degradation and stability 1998, 59 (1-3), 209214.

2. Duran, H.; Steinhart, M.; Butt, H.-J. r.; Floudas, G., From heterogeneous to homogeneous nucleation of isotactic poly (propylene) confined to nanoporous alumina. Nano letters 2011, 11 (4), 1671-1675.

3. Suzuki, Y.; Duran, H.; Steinhart, M.; Butt, H.-J.; Floudas, G., Homogeneous crystallization and local dynamics of poly (ethylene oxide)(PEO) confined to nanoporous alumina. Soft Matter 2013, 9 (9), 2621-2628.

4. Suzuki, Y.; Duran, H.; Akram, W.; Steinhart, M.; Floudas, G.; Butt, H.-J., Multiple nucleation events and local dynamics of poly ( $\varepsilon$-caprolactone)(PCL) confined to nanoporous alumina. Soft Matter 2013, 9 (38), 9189-9198.

5. Suzuki, Y.; Duran, H.; Steinhart, M.; Butt, H.-J. r.; Floudas, G., Suppression of Poly (ethylene oxide) Crystallization in Diblock Copolymers of Poly (ethylene oxide)-b-poly ( $\varepsilon$ caprolactone) Confined to Nanoporous Alumina. Macromolecules 2014, 47 (5), 1793-1800.

6. Jiang, Q.; Ward, M. D., Crystallization under nanoscale confinement. Chemical Society Reviews 2014, 43 (7), 2066-2079. 
7. Wu, H.; Higaki, Y.; Takahara, A., Molecular self-assembly of one-dimensional polymer nanostructures in nanopores of anodic alumina oxide templates. Progress in Polymer Science 2018, 77, 95-117.

8. Politidis, C.; Alexandris, S.; Sakellariou, G.; Steinhart, M.; Floudas, G., Dynamics of Entangled cis-1, 4-Polyisoprene Confined to Nanoporous Alumina. Macromolecules 2019.

9. Mijangos, C.; Hernandez, R.; Martin, J., A review on the progress of polymer nanostructures with modulated morphologies and properties, using nanoporous AAO templates. Progress in Polymer Science 2016, 54, 148-182.

10. Alexandris, S.; Sakellariou, G.; Steinhart, M.; Floudas, G., Dynamics of unentangled cis1, 4-polyisoprene confined to nanoporous alumina. Macromolecules 2014, 47 (12), 3895-3900.

11. Alexandris, S.; Papadopoulos, P.; Sakellariou, G.; Steinhart, M.; Butt, H.-J. r.; Floudas, G., Interfacial energy and glass temperature of polymers confined to nanoporous alumina. Macromolecules 2016, 49 (19), 7400-7414.

12. Masuda, H.; Fukuda, K., Ordered metal nanohole arrays made by a two-step replication of honeycomb structures of anodic alumina. science 1995, 268 (5216), 1466-1468.

13. Masuda, H.; Hasegwa, F.; Ono, S., Self-ordering of cell arrangement of anodic porous alumina formed in sulfuric acid solution. Journal of the electrochemical society 1997, 144 (5), L127-L130.

14. Martín, J.; Manzano, C. V.; Martín-González, M., In-depth study of self-ordered porous alumina in the 140-400 nm pore diameter range. Microporous and Mesoporous Materials 2012, 151, 311-316.

15. Martín, J.; Manzano, C. V.; Caballero-Calero, O.; Martín-González, M., High-aspect-ratio and highly ordered 15-nm porous alumina templates. ACS applied materials \& interfaces 2012, 5 (1), 72-79.

16. Manzano, C. V.; Martín, J.; Martín-González, M. S., Ultra-narrow 12 nm pore diameter self-ordered anodic alumina templates. Microporous and Mesoporous Materials 2014, 184, 177183.

17. Guan, Y.; Liu, G.; Gao, P.; Li, L.; Ding, G.; Wang, D., Manipulating crystal orientation of poly (ethylene oxide) by nanopores. ACS Macro Letters 2013, 2 (3), 181-184.

18. Shi, G.; Liu, G.; Su, C.; Chen, H.; Chen, Y.; Su, Y.; Müller, A. J.; Wang, D., Reexamining the crystallization of poly ( $\varepsilon$-caprolactone) and isotactic polypropylene under hard confinement: nucleation and orientation. Macromolecules 2017, 50 (22), 9015-9023.

19. Martín, J.; Mijangos, C.; Sanz, A.; Ezquerra, T. A.; Nogales, A., Segmental dynamics of semicrystalline poly (vinylidene fluoride) nanorods. Macromolecules 2009, 42 (14), 5395-5401.

20. Steinhart, M.; Senz, S.; Wehrspohn, R. B.; Gösele, U.; Wendorff, J. H., Curvature-directed crystallization of poly (vinylidene difluoride) in nanotube walls. Macromolecules 2003, 36 (10), 3646-3651.

21. Steinhart, M.; Göring, P.; Dernaika, H.; Prabhukaran, M.; Gösele, U.; Hempel, E.; ThurnAlbrecht, T., Coherent kinetic control over crystal orientation in macroscopic ensembles of polymer nanorods and nanotubes. Physical review letters 2006, 97 (2), 027801.

22. Guan, Y.; Liu, G.; Ding, G.; Yang, T.; Müller, A. J.; Wang, D., Enhanced crystallization from the glassy state of poly (L-lactic acid) confined in anodic alumina oxide nanopores. Macromolecules 2015, 48 (8), 2526-2533.

23. Dai, X.; Li, H.; Ren, Z.; Russell, T. P.; Yan, S.; Sun, X., Confinement Effects on the Crystallization of Poly (3-hydroxybutyrate). Macromolecules 2018, 51 (15), 5732-5741. 
24. Casas, M. T.; Michell, R. M.; Blaszczyk-Lezak, I.; Puiggali, J.; Mijangos, C.; Lorenzo, A. T.; Müller, A. J., Self-assembly of semicrystalline PE-b-PS diblock copolymers within AAO nanoporous templates. Polymer 2015, 70, 282-289.

25. Wu, H.; Wang, W.; Yang, H.; Su, Z., Crystallization and orientation of syndiotactic polystyrene in nanorods. Macromolecules 2007, 40 (12), 4244-4249.

26. Wu, H.; Higaki, Y.; Nojima, S.; Takahara, A., Orientation and crystallization of regioregular poly (3-dodecylthiophene) in alumina nanopores. Soft Matter 2017, 13 (26), 46614666.

27. Yu, S.; Lai, Z.; Jinnai, H.; Zeng, X.; Ageishi, M.; Lotz, B.; Cheng, S. Z.; Zheng, N.; Zhang, S.; Feng, X., Adding Symmetry: Cylindrically Confined Crystallization of Nylon-6. Macromolecules 2019.

28. Michell, R. M.; Blaszczyk-Lezak, I.; Mijangos, C.; Müller, A. J., Confinement effects on polymer crystallization: From droplets to alumina nanopores. Polymer 2013, 54 (16), 4059-4077. 29. Michell, R. M.; Müller, A. J., Confined crystallization of polymeric materials. Progress in Polymer Science 2016, 54, 183-213.

30. Michell, R. M.; Blaszczyk-Lezak, I.; Mijangos, C.; Müller, A. J., Confined crystallization of polymers within anodic aluminum oxide templates. Journal of Polymer Science Part B: Polymer Physics 2014, 52 (18), 1179-1194.

31. Schlegel, I.; Muñoz-Espí, R.; Renz, P.; Lieberwirth, I.; Floudas, G.; Suzuki, Y.; Crespy, D.; Landfester, K., Crystallinity tunes permeability of polymer nanocapsules. Macromolecules 2017, 50 (12), 4725-4732.

32. Su, C.; Shi, G.; Li, X.; Zhang, X.; Müller, A. J.; Wang, D.; Liu, G., Uniaxial and Mixed Orientations of Poly (ethylene oxide) in Nanoporous Alumina Studied by X-ray Pole Figure Analysis. Macromolecules 2018, 51 (23), 9484-9493.

33. Müller, A. J.; Balsamo, V.; Arnal, M. L., Nucleation and crystallization in diblock and triblock copolymers. In Block Copolymers II, Springer: 2005; pp 1-63.

34. Hoffman, J. D., Anelastic and dielectric effects in polymeric solids. By NG McCrum, BE Read, and G. Williams, Wiley, 1967, pp. 617, \$25.00, Lib. Congress Card 67: 29334. Journal of Polymer Science B Polymer Physics 1969, 7, 750-750.

35. Schönhals, A.; Kremer, F., Analysis of dielectric spectra. In Broadband dielectric spectroscopy, Springer: 2003; pp 59-98.

36. Martin, J.; Krutyeva, M.; Monkenbusch, M.; Arbe, A.; Allgaier, J.; Radulescu, A.; Falus, P.; Maiz, J.; Mijangos, C.; Colmenero, J., Direct observation of confined single chain dynamics by neutron scattering. Physical review letters 2010, 104 (19), 197801.

37. Blaszczyk-Lezak, I.; Hernández, M.; Mijangos, C., One dimensional PMMA nanofibers from AAO templates. Evidence of confinement effects by dielectric and Raman analysis. Macromolecules 2013, 46 (12), 4995-5002.

38. Martin, J.; Maiz, J.; Sacristan, J.; Mijangos, C., Tailored polymer-based nanorods and nanotubes by" template synthesis": From preparation to applications. Polymer 2012, 53 (6), 11491166.

39. Jessensky, O.; Müller, F.; Gösele, U., Self-organized formation of hexagonal pore structures in anodic alumina. Journal of the Electrochemical Society 1998, 145 (11), 3735-3740. 40. Socrates, G., Infrared and Raman characteristic group frequencies: tables and charts. John Wiley \& Sons: 2004.

41. Tammer, M., G. Sokrates: Infrared and Raman characteristic group frequencies: tables and charts. Springer: 2004. 
42. Zhao, J. B.; Li, K. Y.; Yang, W. T., Chain extension of polybutylene adipate and polybutylene succinate with adipoyl-and terephthaloyl-biscaprolactamate. Journal of applied polymer science 2007, 106 (1), 590-598.

43. Abderrahim, B.; Abderrahman, E.; Mohamed, A.; Fatima, T.; Abdesselam, T.; Krim, O., Kinetic thermal degradation of cellulose, polybutylene succinate and a green composite: comparative study. World Journal of Environmental Engineering 2015, 3 (4), 95.

44. Wang, H.; Ji, J.; Zhang, W.; Zhang, Y.; Jiang, J.; Wu, Z.; Pu, S.; Chu, P. K., Biocompatibility and bioactivity of plasma-treated biodegradable poly (butylene succinate). Acta biomaterialia 2009, 5 (1), 279-287.

45. Yan, C.; Zhang, Y.; Hu, Y.; Ozaki, Y.; Shen, D.; Gan, Z.; Yan, S.; Takahashi, I., Melt crystallization and crystal transition of poly (butylene adipate) revealed by infrared spectroscopy. The Journal of Physical Chemistry B 2008, 112 (11), 3311-3314.

46. Debuissy, T.; Pollet, E.; Avérous, L., Synthesis and characterization of biobased poly (butylene succinate-ran-butylene adipate). Analysis of the composition-dependent physicochemical properties. European Polymer Journal 2017, 87, 84-98.

47. Huang, C. Q.; Luo, S. Y.; Xu, S. Y.; Zhao, J. B.; Jiang, S. L.; Yang, W. T., Catalyzed chain extension of poly (butylene adipate) and poly (butylene succinate) with 2, 2'-(1, 4phenylene)-bis (2-oxazoline). Journal of applied polymer science 2010, 115 (3), 1555-1565.

48. Partini, M.; Pantani, R., FTIR analysis of hydrolysis in aliphatic polyesters. Polymer degradation and stability 2007, 92 (8), 1491-1497.

49. Li, F.; Zhang, L.; Metzger, R. M., On the growth of highly ordered pores in anodized aluminum oxide. Chemistry of materials 1998, 10 (9), 2470-2480.

50. Sousa, C.; Leitao, D.; Proenca, M.; Ventura, J.; Pereira, A.; Araujo, J., Nanoporous alumina as templates for multifunctional applications. Applied Physics Reviews 2014, 1 (3), 031102.

51. Mi, C.; Zhou, J.; Ren, Z.; Li, H.; Sun, X.; Yan, S., The phase transition behavior of poly (butylene adipate) in the nanoporous anodic alumina oxide. Polymer Chemistry 2016, 7 (2), 410417.

52. Shimizu, T., Self-assembled nanomaterials II: nanotubes. Springer: 2008; Vol. 220.

53. Shi, G.; Liu, G.; Su, C.; Chen, H.; Chen, Y.; Su, Y.; Müller, A. J.; Wang, D., Reexamining the crystallization of poly ( $\varepsilon$-caprolactone) and isotactic polypropylene under hard confinement: nucleation and orientation. Macromolecules 2017, 50 (22), 9015-9023.

54. Di Lorenzo, M. L.; Androsch, R.; Righetti, M. C., Low-temperature crystallization of poly (butylene succinate). European Polymer Journal 2017, 94, 384-391.

55. Yoo, E.; Im, S., Melting behavior of poly (butylene succinate) during heating scan by DSC. Journal of Polymer Science Part B: Polymer Physics 1999, 37 (13), 1357-1366.

56. Liu, G.; Zheng, L.; Zhang, X.; Li, C.; Jiang, S.; Wang, D., Reversible lamellar thickening induced by crystal transition in poly (butylene succinate). Macromolecules 2012, 45 (13), 54875493.

57. Yang, J.; Pan, P.; Hua, L.; Xie, Y.; Dong, T.; Zhu, B.; Inoue, Y.; Feng, X., Fractionated crystallization, polymorphic crystalline structure, and spherulite morphology of poly (butylene adipate) in its miscible blend with poly (butylene succinate). Polymer 2011, 52 (15), 3460-3468. 58. Wang, M.; Tashiro, K.; Ozaki, Y., Reinvestigation of the $\beta$-to- $\alpha$ Crystal Phase Transition of Poly (butylene adipate) by the Time-Resolved X-ray Scattering and FTIR Spectral Measurements in the Temperature-Jump Process. Macromolecules 2017, 50 (10), 3883-3889.

59. Sun, X.; Fang, Q.; Li, H.; Ren, Z.; Yan, S., Effect of anodic alumina oxide pore diameter on the crystallization of poly (butylene adipate). Langmuir 2016, 32 (13), 3269-3275. 
60. Minke, R.; Blackwell, J., Single crystals of poly (tetramethylene adipate). Journal of Macromolecular Science, Part B: Physics 1980, 18 (2), 233-255.

61. Minke, R.; Blackwell, J., Polymorphic structures of poly (tetramethylene adipate). Journal of Macromolecular Science, Part B: Physics 1979, 16 (3), 407-417.

62. Pouget, E.; Almontassir, A.; Casas, M. T.; Puiggalí, J., On the crystalline structures of poly (tetramethylene adipate). Macromolecules 2003, 36 (3), 698-705.

63. Polanyi, M., The X-ray fiber diagram. $Z$ Phys 1921, 7, 149-180.

64. Iwata, T.; Kobayashi, S.; Tabata, K.; Yonezawa, N.; Doi, Y., Crystal Structure, Thermal Behavior and Enzymatic Degradation of Poly (tetramethylene adipate) Solution-Grown ChainFolded Lamellar Crystals. Macromolecular bioscience 2004, 4 (3), 296-307.

65. Kremer, F.; Schönhals, A., Broadband dielectric spectroscopy. Springer 2003.

66. Arandia, I.; Mugica, A.; Zubitur, M.; Mincheva, R.; Dubois, P.; Müller, A. J.; Alegría, A., The complex amorphous phase in poly (butylene succinate-ran-butylene azelate) isodimorphic copolyesters. Macromolecules 2017, 50 (4), 1569-1578.

67. Charlon, S.; Delbreilh, L.; Dargent, E.; Follain, N.; Soulestin, J.; Marais, S., Influence of crystallinity on the dielectric relaxations of poly (butylene succinate) and poly [(butylene succinate)-co-(butylene adipate)]. European Polymer Journal 2016, 84, 366-376.

68. Tarnacka, M.; Talik, A.; Kamińska, E.; Geppert-Rybczyńska, M.; Kaminski, K.; Paluch, M., The Impact of Molecular Weight on the Behavior of Poly(propylene glycol) Derivatives Confined within Alumina Templates. Macromolecules 2019, 52 (9), 3516-3529.

69. Li, L.; Zhou, D.; Huang, D.; Xue, G., Double glass transition temperatures of poly (methyl methacrylate) confined in alumina nanotube templates. Macromolecules 2013, 47 (1), 297-303.

70. Li, L.; Chen, J.; Deng, W.; Zhang, C.; Sha, Y.; Cheng, Z.; Xue, G.; Zhou, D., Glass transitions of poly (methyl methacrylate) confined in nanopores: conversion of three-and twolayer models. The Journal of Physical Chemistry B 2015, 119 (15), 5047-5054.

71. Talik, A.; Tarnacka, M.; Grudzka-Flak, I.; Maksym, P.; Geppert-Rybczynska, M.; Wolnica, K.; Kaminska, E.; Kaminski, K.; Paluch, M., The Role of Interfacial Energy and Specific Interactions on the Behavior of Poly (propylene glycol) Derivatives under 2D Confinement. Macromolecules 2018, 51 (13), 4840-4852.

72. Tarnacka, M.; Kaminski, K.; Mapesa, E. U.; Kaminska, E.; Paluch, M., Studies on the Temperature and Time Induced Variation in the Segmental and Chain Dynamics in Poly (propylene glycol) Confined at the Nanoscale. Macromolecules 2016, 49 (17), 6678-6686. 
For Table of Contents Use Only

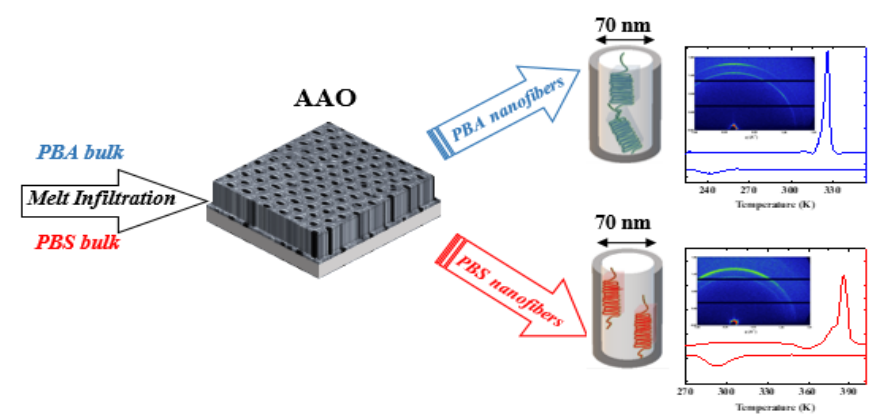

